\title{
Low-frequency electrostatic waves in the ionospheric E-region: a comparison of rocket observations and numerical simulations
}

\author{
L. Dyrud ${ }^{1,2}$, B. Krane ${ }^{3}$, M. Oppenheim ${ }^{4}$, H. L. Pécseli ${ }^{2,5}$, K. Schlegel ${ }^{6}$, J. Trulsen ${ }^{2,7}$, and A. W. Wernik ${ }^{2.8}$ \\ ${ }^{1}$ Center for Remote Sensing, 3702 Pender Dr., Fairfax, VA 22030, USA \\ ${ }^{2}$ Centre for Advanced Study, Drammensveien 78, N-0271 Oslo, Norway \\ ${ }^{3}$ Norwegian Defense Research Establishment (NDRE), Box 25, N-2027 Kjeller, Norway \\ ${ }^{4}$ Center for Space Physics, Boston University, 725 Commonwealth Ave., Boston MA 02215, USA \\ ${ }^{5}$ University of Oslo, Physics Department, P.O. Boks 1048 Blindern, N-0316 Oslo, Norway \\ ${ }^{6}$ Max Planck Institute für Sonnensystemforschung, D-37191 Katlenburg-Lindau, Germany \\ ${ }^{7}$ University of Oslo, Institute of Theoretical Astrophysics, P.O. Boks 1029 Blindern, N-0315 Oslo, Norway \\ ${ }^{8}$ Space Research Center, Polish Academy of Sciences, ul. Bartycka 18a, 00-716 Warsaw, Poland
}

Received: 20 June 2006 - Accepted: 18 September 2006 - Published: 21 November 2006

\begin{abstract}
Low frequency electrostatic waves in the lower parts of the ionosphere are studied by a comparison of observations by instrumented rockets and of results from numerical simulations. Particular attention is given to the spectral properties of the waves. On the basis of a good agreement between the observations and the simulations, it can be argued that the most important nonlinear dynamics can be accounted for in a 2-D numerical model, referring to a plane perpendicular to a locally homogeneous magnetic field. It does not seem necessary to take into account turbulent fluctuations or motions in the neutral gas component. The numerical simulations explain the observed strongly intermittent nature of the fluctuations: secondary instabilities develop on the large scale gradients of the largest amplitude waves, and the small scale dynamics is strongly influenced by these secondary instabilities. We compare potential variations obtained at a single position in the numerical simulations with two point potential-difference signals, where the latter is the adequate representation for the data obtained by instrumented rockets. We can demonstrate a significant reduction in the amount of information concerning the plasma turbulence when the latter signal is used for analysis. In particular we show that the bicoherence estimate is strongly affected. The conclusions have implications for studies of low frequency ionospheric fluctuations in the $\mathrm{E}$ and $\mathrm{F}$ regions by instrumented rockets, and also for other methods relying on difference measurements, using two probes with large separation. The analysis also resolves a long standing controversy concerning the supersonic phase velocities of these cross-field instabilities being observed in laboratory experiments.
\end{abstract}

Correspondence to: H. L. Pécseli

(hans.pecseli@fys.uio.no)
Keywords. Ionosphere (Ionospheric irregularities) - Space plasma physics (Numerical simulation studies; Turbulence)

\section{Introduction}

Low frequency electrostatic fluctuations are frequently observed in the lower parts of the Earth's ionospheric E-region, in Equatorial as well as in the polar ionospheres. Several candidates for instabilities giving rise to these waves have been proposed (Rogister and D’Angelo, 1970). For the present analysis, we emphasize the instability that arises in a plasma with a large ion-neutral collision frequency, $v_{i}>\Omega_{c i}$, while simultaneously the opposite inequality holds for the electron collisions, $v_{e} \ll \Omega_{c e}$. The source of free energy in the system is a dc-electric field imposed in the direction perpendicular to the Earth's magnetic field (Farley, 1963; Buneman, 1963). These fluctuations were originally discovered by radar scattering off the ionosphere, and later investigated by in-situ measurements by instrumented rockets. In a sense the rocket and the radar represent complementary types of diagnostics: the radar selects a constant wavelength determined by the wavenumber matching condition, while the rocket data are evidently dominated by the largest amplitude signal, irrespective of its characteristic wavelength.

For later reference we give here a simplified version of the linear dispersion relation obtained by a fluid plasma model. The real and imaginary parts of the frequency are denoted $\omega_{r}$ and $\omega_{i}$, respectively.

$$
\begin{aligned}
\omega_{r} & =\frac{k V_{d} \cos \alpha}{1+\varphi}, \\
\omega_{i} & =\frac{\varphi}{1+\varphi}\left(\frac{1}{v_{i}}\left(\omega_{r}^{2}-k^{2} C_{s}^{2}\right)+\frac{\omega_{r} \Omega_{c e}}{k L_{n} \nu_{e}}\right),
\end{aligned}
$$


where

$\varphi=\frac{v_{e} v_{i}}{\Omega_{c e} \Omega_{c i}}\left(1+\frac{\Omega_{c e}^{2} k_{\|}^{2}}{v_{e}^{2} k^{2}}\right)$,

and $L_{n}$ denotes the scale length of a possible large scale plasma density gradient in the direction $\perp \mathbf{B}$, while $V_{d}$ is the difference between the electron-ion drift velocities, and $\alpha$ is the angle between $\mathbf{V}_{d}$ and $\mathbf{k}$. The analysis uses the quasineutrality assumption, and consequently the results only apply for wavelengths much longer than the Debye length, $\lambda_{D}$. The results (1)-(2) are valid in the limit of very small growth rates, $\omega_{i} \ll \omega_{r}$, and almost $\mathbf{B}$-perpendicular wave propagations, $k_{\|} \ll k_{\perp}$. We note that a gradient in plasma density contributes to an instability at any drift velocity (last term in Eq. 2). We will argue that for the relevant plasma conditions analyzed in the following, we can ignore large scale plasma density gradients $\perp \mathbf{B}$. The relative drift between electrons and ions has to exceed the ion sound speed $C_{s}$ in order to give unstable waves, otherwise it has a damping effect. In this simple model, the first waves to become unstable are those where $\mathbf{k} \perp \mathbf{B}$. Since $\Omega_{c e} \gg v_{e}$ and $\Omega_{c i} \leq v_{i}$ for the relevant ionospheric conditions, we find that waves with large $k_{\|}$give large $\varphi$ and therefore small $\omega_{r}$, and will consequently remain linearly stable for realistic values of $V_{d}$. Recombination acts as a damping mechanism in all cases, but it is not included in Eqs. (1)-(2).

The basic features of the Farley-Buneman instability are well understood, but a number of features are difficult to account for, in particular concerning a disagreement between the observations and results from several laboratory investigation (D'Angelo et al., 1974; John and Saxena, 1975; Mikkelsen and Pécseli, 1980). Several nonlinear saturation models have been suggested (Sudan, 1983; Primdahl and Bahnsen, 1985; Primdahl, 1986; Hamza and StMaurice, 1995), to account for the deviations between spaceobservation and what a simple extrapolation from the linear theoretical analysis seems to predict.

One serious problem associated with the interpretation of the data is the lack of information concerning the detailed state of the ionospheric plasma: the rockets are usually equipped with sensors for three components of the electric fields, one or two Faraday-cups for density (or, rather, current), but usually only little is known concerning the neutral background. Also, the rocket samples the ionospheric irregularities along a simple trajectory, and it is not in all cases evident how to distinguish a stationary structure in plasma density from one associated with the propagating waves. It is difficult to determine to what extent stationary plasma density striations are present, and how important they are for the plasma dynamics. As far as striations in the neutral component are concerned, in the form of, for instance, wind-shear, the question is even more difficult since the rockets have no instruments to detect such phenomena. It might very well be that the neutral background component is in a turbulent state
(Thrane et al., 1981), and this has been suggested to be of importance for the spectral evolution of the plasma turbulence (Schlegel and Gurevich, 1997).

In the present study we will analyze data from instrumented rockets, as obtained from the ROSE campaign (Rose et al., 1992), and compare the results with those obtained by direct numerical simulations. We use data obtained by detecting the potential difference between two probes. Also data for fluctuating plasma density is available, but these have been analyzed elsewhere (Iranpour et al., 1997; Krane et al., 2000), and are not discussed here.

Numerical simulations have proved to be valuable tools for analyzing some of the properties of the saturated waves (Oppenheim et al., 1995; Oppenheim and Otani, 1996). We carry out the numerical simulations for ideal conditions, without plasma density striations and inhomogeneities in the neutral velocity field.

The value of the electron temperature is important for the modeling, and we included in Appendix A discussions of this question. In Appendix B we have a short discussion which is addressing synthetic data modeling of wave phenomena as those discussed in the present paper.

\section{Ionospheric rocket data}

During the ROSE rocket campaign, four instrumented payloads were launched altogether, F1 and F2 in NovemberDecember 1988 from Andøya, Norway, and F3 and F4 in February 1989 from Kiruna, Sweden. Peak altitudes were in the range $115-125 \mathrm{~km}$. In the present study we analyze data from the F4 experiment. The ROSE4 rocket was launched in a direction perpendicular to the Hall current of the electrojet, see Fig. 1 for details. The magnetic field lines are here almost perpendicular to ground, the dip angle being approximately $77^{\circ}$. The local drift vectors shown on Fig. 1 are obtained from the rocket data by assuming an $\mathbf{E}_{0} \times \mathbf{B}$ drift. The circle with an arrow gives an independent measurement of the direction of the plasma drift, as obtained by the European Incoherent Scatter radar (EISCAT) at the indicated altitude (Rinnert, 1992). The length of that arrow corresponds to a deduced electric field of $45 \mathrm{mV} / \mathrm{m}$. Comparison with STARE-data are presented by Rinnert (1992). The orientation of the rocket is essentially constant during the flight, since the cone-angle is small. The angle between the angular momentum vector of the rocket and ground can be take to be a constant, $67^{\circ}$. The trajectory on the downleg part is almost parallel to the local magnetic field lines, see also Fig. 1.

The ELF signals analyzed here were obtained by means of gold-plated spherical probes of $5 \mathrm{~cm}$ diameter, mounted on two pairs of booms, one near the top of the payload (labeled 1 and 2) and the other $185 \mathrm{~cm}$ lower (labeled 3 and 4), oriented at an angle of $90^{\circ}$ with respect to the first pair, as illustrated in Fig. 2 (Rinnert, 1992). The length of each boom was $180 \mathrm{~cm}$, giving a probe separation 


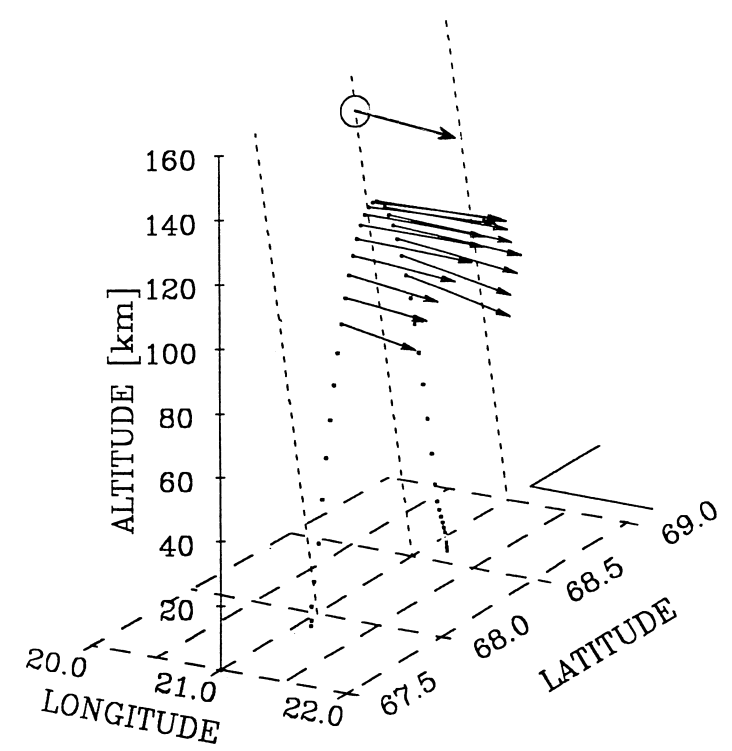

Fig. 1. Diagrammatic representation of the ROSE4 rocket trajectory, shown by the dots (Rinnert, 1992). The dashed lines give the direction of the magnetic field. The apogee of this rocket was $123 \mathrm{~km}$. Arrows indicate $\mathbf{E}_{0} \times \mathbf{B}$-drifts.

of $360 \mathrm{~cm}$ on each boom. We analyzed the fluctuating signals $U_{6}(t)=\phi_{1}(t)-\phi_{2}(t) ; U_{5}(t)=\phi_{4}(t)-\phi_{3}(t) ; U_{4}(t)$ $=\phi_{1}(t)-\phi_{4}(t) ; U_{3}(t)=\phi_{2}(t)-\phi_{3}(t) ; U_{2}(t)=\phi_{1}(t)-\phi_{3}(t)$; and $U_{1}(t)=\phi_{2}(t)-\phi_{4}(t)$, where $\phi_{j}(t)$ for $j=1,2,3,4 \mathrm{de}-$ notes the potential on the $j$-th probe with respect to a suitably defined common ground. There is an evident redundancy in the available signals, which can be used to check the performance of individual probes. For wavelengths much larger than the probe separations, it is evident that the potential difference signals can be used to estimate the fluctuating electric fields. The space-time varying electric field fluctuations of the electrojet were originally sampled with a $4 \mathrm{kHz}$ sampling frequency. By averaging sampling points two-by-two, we here increase the sampling interval to $0.5 \mathrm{~ms}$, giving a Nyquist frequency of $1000 \mathrm{~Hz}$. The electric circuits give an effective frequency limitation closer to $600 \mathrm{~Hz}$. The signals were digitized with a 12 bit resolution. The dc-electric fields were measured by the same probes. The probes measure floating potential, $\phi_{f}$, in principle. At these low frequencies, we can assume the local potential difference between $\phi_{f}$ and the plasma potential $\phi_{p}$, to be constant, since the plasma conditions are unlikely to change significantly on distances corresponding to the probe separations. Note that ROSE4 was night time launch (at 23:43:00 UT), so that photo-emission differences between probes in sunlight and in the shadow of the rocket body are not an issue here.

The ROSE rockets were also equipped with two retarding potential analyzers, one pointing along the rocket axis (labeled $P_{1}$ in the following) and one in the perpendicular direction (labeled $P_{2}$ ), see also Fig. 2. When properly nor-

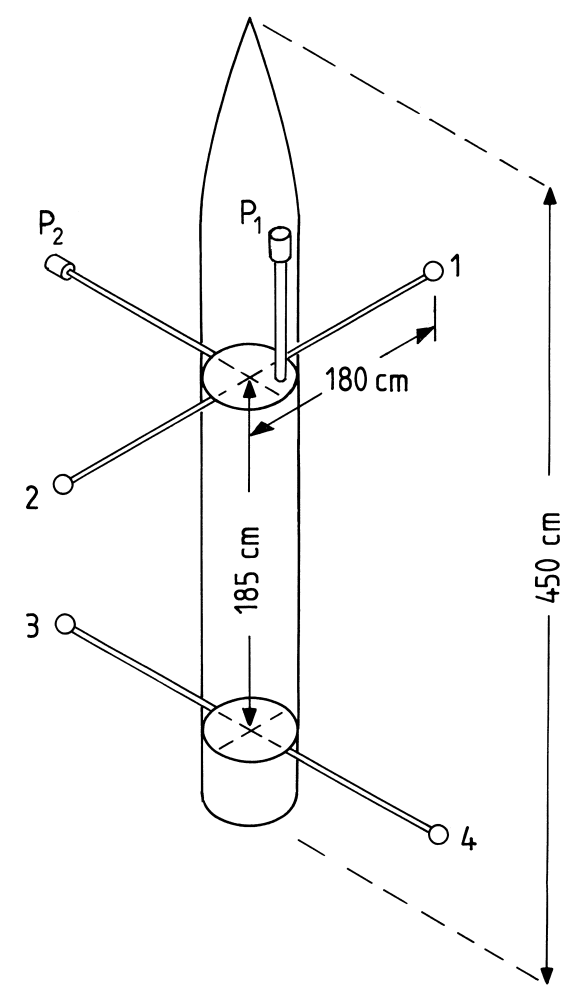

Fig. 2. Schematic diagram for the positioning of the probes and retarding potential analyzers on the ROSE rockets. The rocket is here shown with the nose-cone intact.

malized, the signals from these analyzers can be interpreted as a measure of the fluctuations in plasma density (Schlegel, 1992). These density signals have been studied elsewhere (Krane et al., 2000), and will not be discussed here.

Because of the rocket spin relative to the dc-electric field, the electric field signal has a large-amplitude variation following the rocket spin, with the fluctuating wave component being superimposed. Since these large-amplitude signals at the rocket spin frequency are not being discussed in the present study, we remove the fundamental and first few harmonics of this low-frequency variation by an $8-\mathrm{Hz}$ highpass filtering. The filtering is made digitally, and it does not induce any phase changes in the filtered signal. Quite generally, it can be argued (Pécseli et al., 1989) that the rocket spin gives rise to an amplitude as well as a phase modulation of the ionospheric signals. For the frequency range relevant for the present study, these effects are immaterial.

The ionospheric conditions and details of the instrumentation relevant for the present data set were discussed in a special issue of Journal of Atmospheric and Terrestrial Physics $(54,655-818,1992)$. Here a short summary will suffice: The dc-electric field values of approximately 40 and $70 \mathrm{mV} / \mathrm{m}$ were measured on upleg and downleg passages of the Eregion, respectively, see also Table 1 . The corresponding $\mathbf{E}_{0} \times \mathbf{B} / B^{2}$ velocities are approximately 800 and $1400 \mathrm{~m} / \mathrm{s}$, 
Table 1. E-region plasma and field parameters during the ROSE4 flight.

\begin{tabular}{lcc}
\hline Parameter & Value & Unit \\
\hline $\begin{array}{l}\text { Peak plasma density, } n_{0}, \\
\text { at an altitude of 116 km (Schlegel, 1992) }\end{array}$ & $1.6 \cdot 10^{11}$ & $\mathrm{~m}^{-3}$ \\
Magnetic field, $B_{0}$ & 50 & $\mu \mathrm{T}$ \\
Average upleg DC electric field, $E_{0}$ & 40 & $\mathrm{mV} / \mathrm{m}$ \\
Average upleg value of $E_{0} / B_{0}$ & 800 & $\mathrm{~m} / \mathrm{s}$ \\
Average downleg DC electric field, $E_{0}$ & 70 & $\mathrm{mV} / \mathrm{m}$ \\
Average downleg value of $E_{0} / B_{0}$ & 1400 & $\mathrm{~m} / \mathrm{s}$ \\
Electron temperature, $T_{e}$ & 400 & $\mathrm{~K}$ \\
Ion temperature, $T_{i}$ & 200 & $\mathrm{~K}$ \\
Electron Debye-length, $\lambda_{D}$ & $6 \cdot 10^{-3}$ & $\mathrm{~m}$ \\
Vertical plasma density scale-length & 12.5 & $\mathrm{~km}$ \\
Sound speed & 400 & $\mathrm{~m} / \mathrm{s}$ \\
Electron cyclotron frequency, $\Omega_{e}$ & $9.3 \cdot 10^{6}$ & $\mathrm{rad} / \mathrm{s}$ \\
$\begin{array}{l}\text { Ion cyclotron frequency, } \Omega_{i}, \\
\text { corresponding to an average }\end{array}$ & 180 & $\mathrm{rad} / \mathrm{s}$ \\
mass of 31 amu & & \\
$\begin{array}{l}\text { Ion-neutral collision frequency, } v_{i n}, \\
\text { at an altitude of } 110 \mathrm{~km}\end{array}$ & 600 & $\mathrm{~s}$ \\
$\begin{array}{l}\text { Electron-neutral collision frequency, } v_{e n}, \\
\text { at an altitude of } 110 \mathrm{~km}\end{array}$ & $1.5 \cdot 10^{4}$ & $\mathrm{~s}$ \\
\hline
\end{tabular}

eastward (see also Figs. 3 and 1). These are of a sufficient magnitude to excite the Farley-Buneman instability (Farley, 1963; Buneman, 1963; Rogister and D'Angelo, 1970). We note that also the direction of the dc-electric field changes slightly for downleg conditions, see also Fig. 1. Electrostatic E-field fluctuations with typical rms amplitudes of $4-8 \mathrm{mV} / \mathrm{m}$ were observed in the altitude range $90-110 \mathrm{~km}$ for the flights. The fluctuations decayed slowly for increasing altitudes, eventually to disappear at around $115 \mathrm{~km}$. In Fig. 3 we show altitude variations of calculated collision frequencies, temperatures, and relevant velocities.

The ion temperature is here assumed to be close to that of the neutral component. The neutral air temperature was measured by instruments on the rockets (Friker and Lübken, 1992). The value for the electron temperature is based on a model that is supported by measurements using the European Incoherent Scatter radar (EISCAT) (Kohl et al., 1992). Measurements on the rocket itself place an upper limit for the actual value of electron temperatures, but the precise temperature is otherwise uncertain. Ionospheric parameters relevant for the flight are listed in Table 1. Collision frequencies are deduced from standard tables.

We find it important to emphasize that the electric fields, $\mathrm{dc}$ and ac, are measured by the instruments on the rocket, and not indirectly deduced from flow velocities or similar mea-

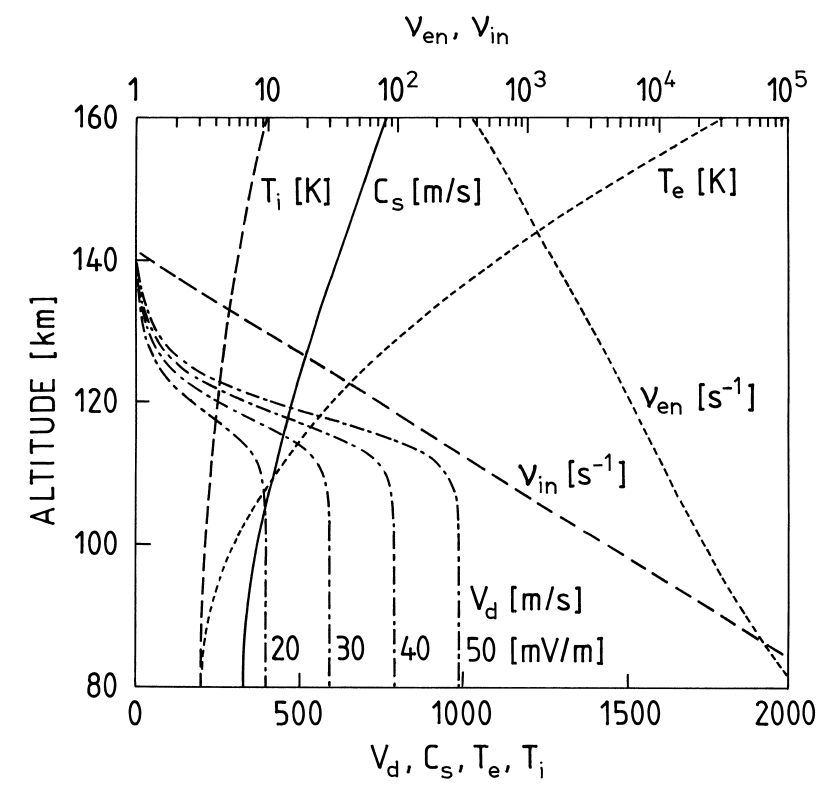

Fig. 3. Altitude variation of collision frequencies, $v_{e}, v_{i}$, and temperatures $T_{e}, T_{i}$, together with the corresponding variation of the sound speed, with $V_{d}$ being the difference between the ion and electron drift velocities calculated for different values of $E_{0}$.

surements. In a different context Rogister (1972) has noted that the electrojet fluctuations can give rise to a large scale polarization electric field, reducing the one imposed on the ionosphere. We measure unambiguously the actual field.

Based on the data from one rocket trajectory alone, we are not able to argue for the presence of gradients in the plasma density in the direction perpendicular to the magnetic field..

\subsection{Analysis of ionospheric data}

We have analyzed parts of the data from the ROSE4 rocket previously (Iranpour et al., 1997; Krane et al., 2000; Larsen et al., 2002) in part by short time Fourier transforms, and also by wavelet-based methods. Several questions are of interest, such as the wave power distribution over frequencies, characteristic propagation velocities and dispersion, where many can be resolved (at least partly) by standard methods. More advanced forms of analysis are required to reveal nonlinear couplings between different frequency components.

We first analyze the direction and velocity of wave propagation, which can be determined relatively accurately by cross-correlation measurements between the signals from probe pairs as $U_{3}$ and $U_{4}$, or $U_{5}$ and $U_{6}$, respectively. The former gives estimates for velocity components in the direction perpendicular to the rocket axis, the latter for the direction along the axis of the rocket. We found that the phasevelocity component along the rocket axis is several times that in the perpendicular direction, indicating (as expected) that the dominant direction of wave propagation is along the electrojet direction, i.e. perpendicular to the rocket axis. By 


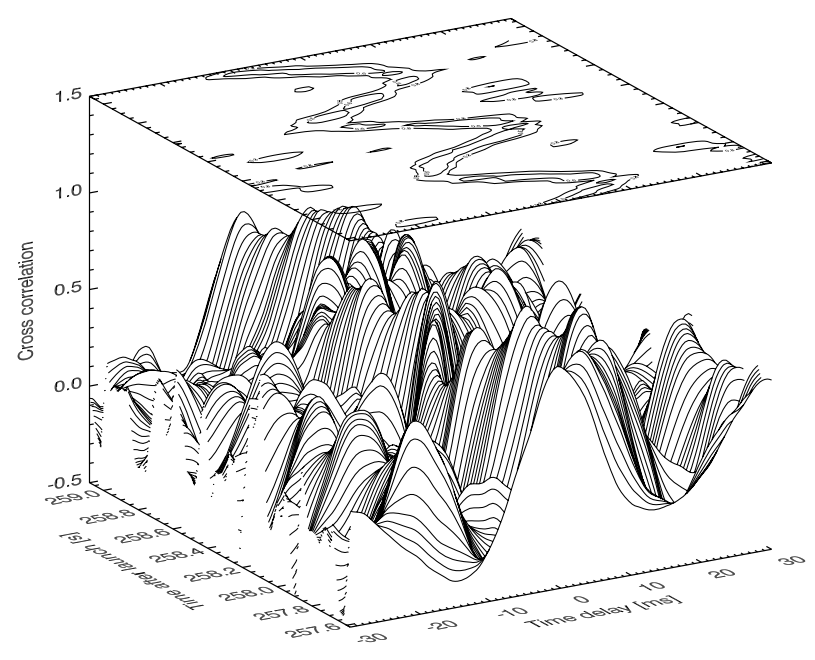

Fig. 4. Sample of local normalized cross-correlation of the signals $U_{1}$ and $U_{2}$ for the time interval $257.58-259.08 \mathrm{~s}$, as indicated. Contour levels are at 0.3 and 0.6. Note that the front curve, with no time delay, corresponds to the local auto-correlation of the signal.

analyzing the cross-correlations of $U_{5}$ and $U_{6}$ and similar combinations, we can estimate the direction and magnitude of the phase velocity of the propagating waves in more detail. The absolute value of the direction can be somewhat uncertain, but the relative variation with time, i.e. altitude, can be obtained more accurately.

If we correlate the signals $U_{1}$ and $U_{2}$ or $U_{3}$ together with $U_{4}$, we obtain information of the velocities in the direction perpendicular to the rocket axis, noting that these two signal combinations are in quadrature, i.e. for propagation strictly perpendicular to the rocket axis the cross correlation of $U_{1}$ and $U_{2}$ is maximally delayed while the $U_{3}-U_{4}$ cross correlation then has vanishing delay and vice versa. The rocket spin will modulate these time-delays as illustrated by the crosscorrelation sample shown in Fig. 4. If we take the maximum delays $\tau_{1}$ and $\tau_{2}$ from these two cross correlations, we can determine two phase velocity components. Labeling, for instance, the wave-numbers in the two directions $k_{1}$ and $k_{2}$, we have that the two time-delays give $\tau_{j}=k_{j} \Delta / \omega$ with $j=1,2$, assuming the waves to be non-dispersive, with $\Delta$ being the probe-pair separation. For the present geometry, see Fig. 2, we have $\Delta=2.54 \mathrm{~m}$. The absolute value of the phase velocity for non-dispersive waves is then at any spin phase of the rocket determined as $u_{p h}=\Delta / \sqrt{\tau_{1}^{2}+\tau_{2}^{2}}$. If the velocity parallel to the rocket axis is comparable to that in the transverse direction, the analytical expressions have to be generalized slightly (Iranpour et al., 1997). The analysis evidently relies on idealizations of the probe geometry, with strictly perpendicular booms etc. The actual conditions of the probes and the booms after launch can not be tested.

The basic results of the analysis are shown in Figs. 5, 6, 7 and 8 where we present the position for the maximum value

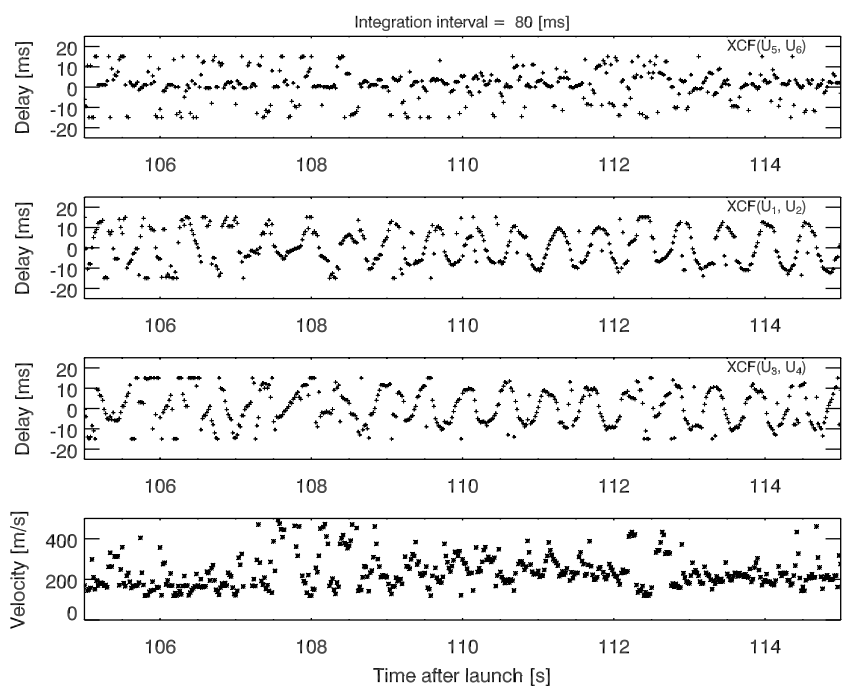

Fig. 5. Top frame shows the time-delay $\tau_{5,6}$ for the maximum of the cross-correlation between $U_{5}(t)$ and $U_{6}(t)$, giving the component for the velocity of propagation along the rocket axis. The two next frames give the corresponding delays for the cross-correlations $U_{1}(t)$ vs. $U_{2}(t)$ and $U_{3}(t)$ vs. $U_{4}(t)$, respectively. Finally, the lowest frame gives the phase velocity component perpendicular to the rocket axis obtained as $\Delta /\left(\tau_{1,2}^{2}+\tau_{3,4}^{2}\right)^{1 / 2}$.

of the cross correlations in question, for selected time intervals in the upleg as well as the downleg parts of the rocket flight. These time-intervals contain the regions of enhanced wave activity. The local cross correlations are obtained using a sliding window of $80 \mathrm{~ms}$, i.e. less than $1 / 6$ of a spin period. Longer windows give a smearing out of the cross correlation, while the scatter on the local maxima increases rapidly for shorter windows. The time-delay of the maximum in the cross-correlations are determined by fitting a continuous function to the sampling points around the local maxima, and the sampling time is therefore not visible (which would otherwise give rise to horizontal "stripes", separated by the sampling time). We note that the clear spin modulation of the time delays support a model where the ionospheric waves are unidirectional, with a well defined velocity component in the direction perpendicular to the axis of the rocket. With the time delays for the cross correlations available and the corresponding phase velocity components as functions of time after launch, we can also construct the altitude variation of the phase velocity in the direction perpendicular to the rocket, as shown in Fig. 9.

On the basis of Figs. 5, 6, 7 and 8, some basic observations are readily made. Thus, we note that the systematically varying time-delays for the propagation along the rocket axis (correlations of $U_{5}(t)$ and $\left.U_{6}(t)\right)$ are very short, usually $1-2 \mathrm{~ms}$, and apart from randomly scattered points, never more than $5 \mathrm{~ms}$. The scatter of points for the time-delays along the rocket axis is relatively large with a small positive 


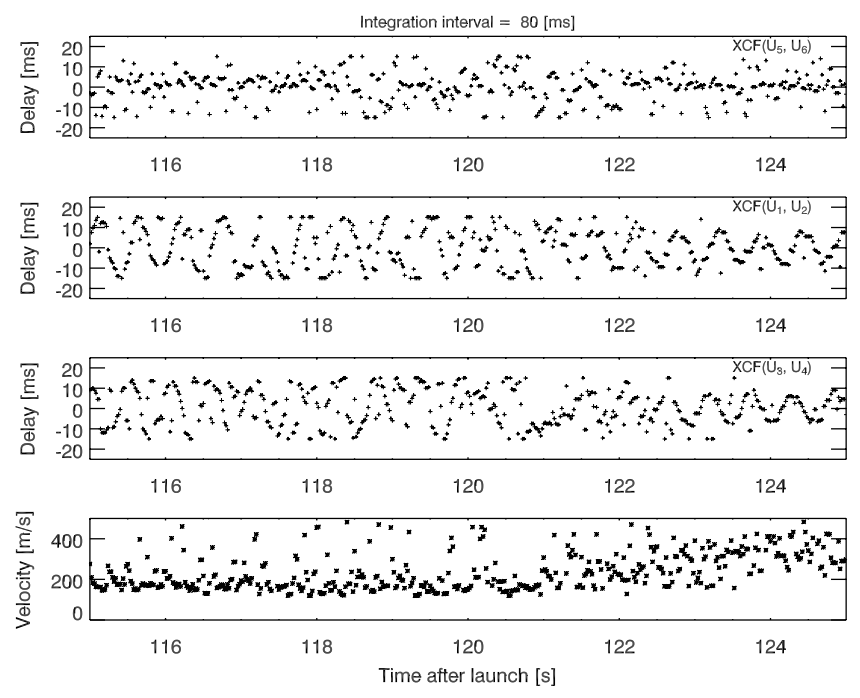

Fig. 6. Cross-correlation results. See Fig. 5 for explanation.

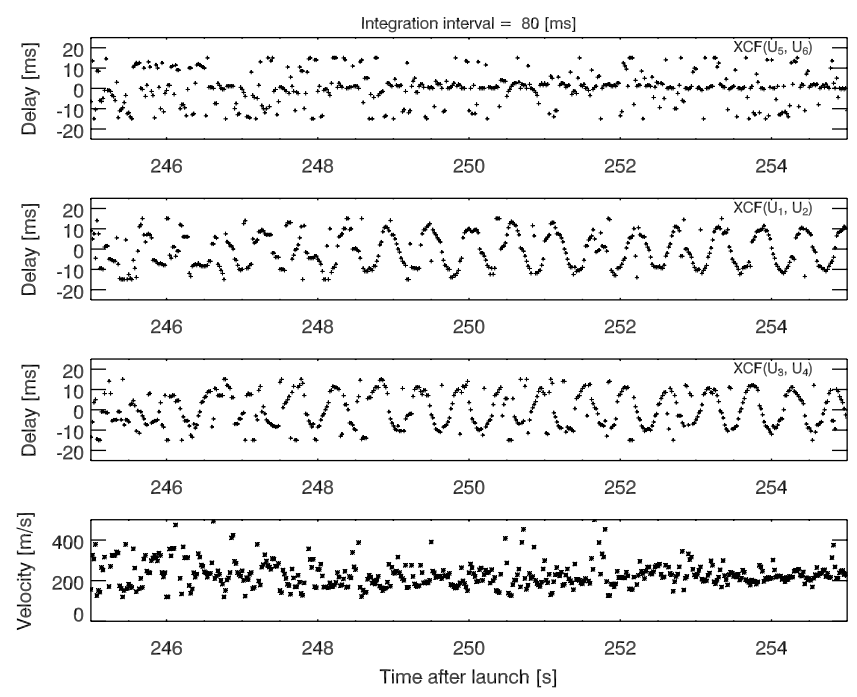

Fig. 7. Cross-correlation results. See Fig. 5 for explanation.

average on the upleg part, while they are significantly more systematic for the downleg parts of the flight. The results indicate that the velocity component of wave-propagation along the rocket axis is very large, $\geq 1 \mathrm{~km} \mathrm{~s}^{-1}$, where we note that a characteristic rocket velocity is $\sim 1 \mathrm{~km} \mathrm{~s}^{-1}$. A more precise analytical approximation for the rocket altitude is $a=-36.41+1.737 t-4.7223 \times 10^{-3} t^{2} \mathrm{~km}$, implying a vertical velocity component $U_{v}=1.737-9.445 \times 10^{-3} t \mathrm{~km} \mathrm{~s}^{-1}$, expressed in time-of-flight $t \mathrm{~s}$. The horizontal velocity component is to the same accuracy $U_{h}=0.196 \mathrm{~km} \mathrm{~s}^{-1}$. The small coning angle of the rocket is ignored here.

The scatter in time delays for the correlations is noticeably smaller on the downleg part of the flight, where the rocket trajectory is almost along $\mathbf{B}$. The observed time delays for the
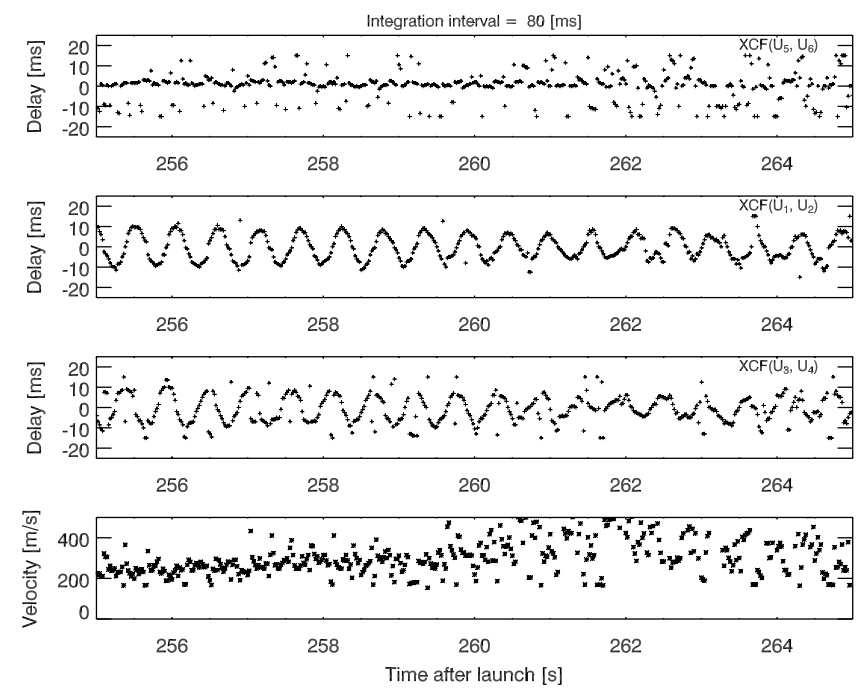

Fig. 8. Cross-correlation results. See Fig. 5 for explanation.

downleg part at times exceeding times-of-flight of $250 \mathrm{~s}$ indicate a predominant direction of propagation from the back (the $U_{5}$-signal) towards the front probe-set (the $U_{6}$-signal). Parts of the signal with a large scatter in observed delays can be interpreted as originating from small stationary, or slowly moving, density perturbations. These will give rise to a timedelay of the order of $1 \mathrm{~ms}$. When interpreting the top frames in Figs. 5, 6, 7 and 8, keep in mind that the spatial orientation of the rocket is fixed during the entire flight, apart from a small coning with a period of approximately $5.8 \mathrm{~s}$. The most conspicuous time-delay in the range of $1-3 \mathrm{~ms}$, see top frames of Figs. 7 and 8 indicate a velocity component of northward propagation of approximately $10^{3} \mathrm{~ms}^{-1}$ in the rocket frame, although the estimate has a significant uncertainty.

It is evident, by inspection of Figs. 5, 6, 7 and 8, that many of the cross-correlation delays can be identified as "spurious", i.e. obviously caused by uncertainties introduced by the short time-sequences. The scatter of the points in Fig. 9 could be reduced by a careful selection, but we see little point in doing so.

With the two phase velocity components available, we can determine also the direction of wave propagation with respect to the rocket axis as $\Phi(t)=\operatorname{ArcTan}\left(\tau_{1} / \tau_{2}\right)$. Results are summarized in Figs. 10 and 11 as functions of time after launch. We subtracted the time variation of the a priori known spin phase of the rocket from $\Phi(t)$. The altitude variation of this phase difference is shown in Fig. 12. We note a systematic variation, which is consistent for the upleg and downleg conditions, indicating a change in direction of dominant wave-propagation with altitude of approximately $0.4 \mathrm{rad}$, or approximately $20^{\circ}$. The "stripes" in Figs. 10 and 11 separated by $\pi$ are caused by the ArcTan-calculation used here: it gives an output in the range $\{-\pi ; \pi\}$. The rocket 

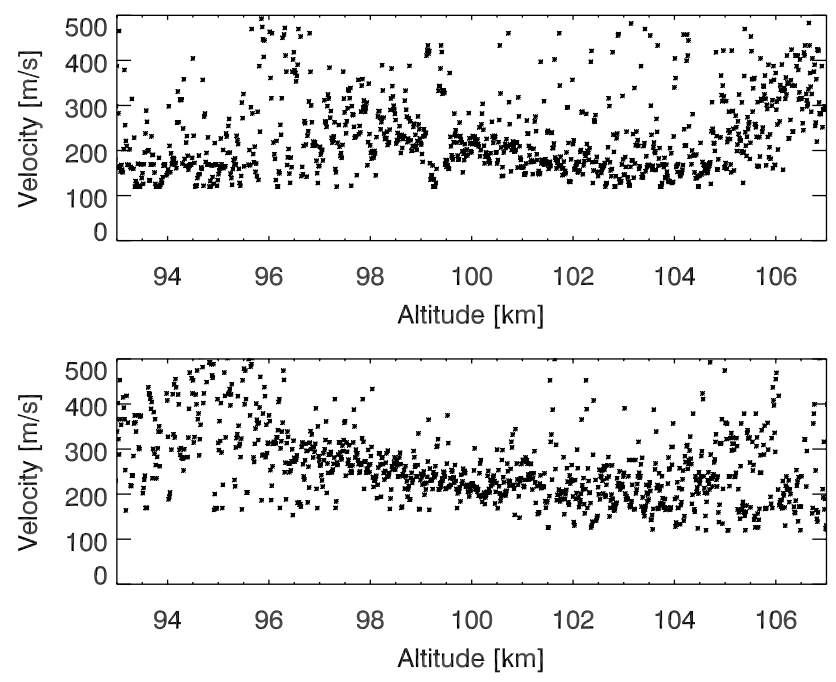

Fig. 9. Altitude variation of phase velocity component perpendicular to the rocket axis for upleg (top) and downleg (bottom) conditions.

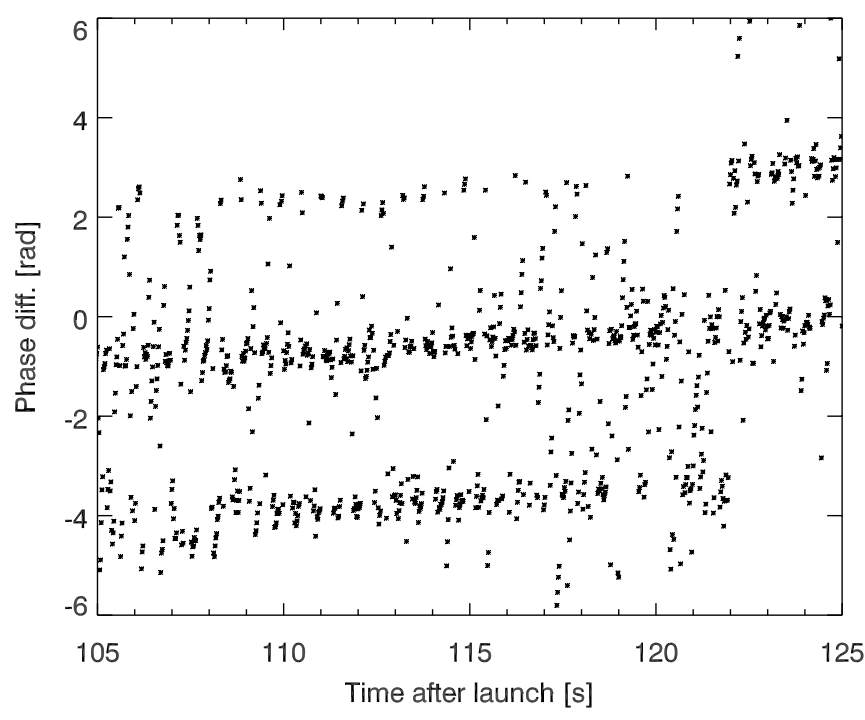

Fig. 10. Relative variation in direction of propagation for upleg conditions.

phase has an almost linear variation with time, and results in a nearly "saw-tooth" variation, when we take $\operatorname{Mod}[2 \pi]$. The directional spin-phase of the waves is sometimes lagging, sometimes advancing slightly, as compared to that of the rocket spin, and the difference between the two can jump between $\pm \pi$ for a short time interval, simply due to small fluctuations in these relative phases. In Fig. 12 we show only the part around zero phase difference.

For reference we recall the collisional dependence of the Hall ion drift as $\left(E_{0} / B\right) /\left(1+\left(v_{i} / \Omega_{c i}\right)^{2}\right)$, while we have $\left(E_{0} / B\right)\left(v_{i} / \Omega_{c i}\right) /\left(1+\left(v_{i} / \Omega_{c i}\right)^{2}\right)$ for the ion Pedersen drift,

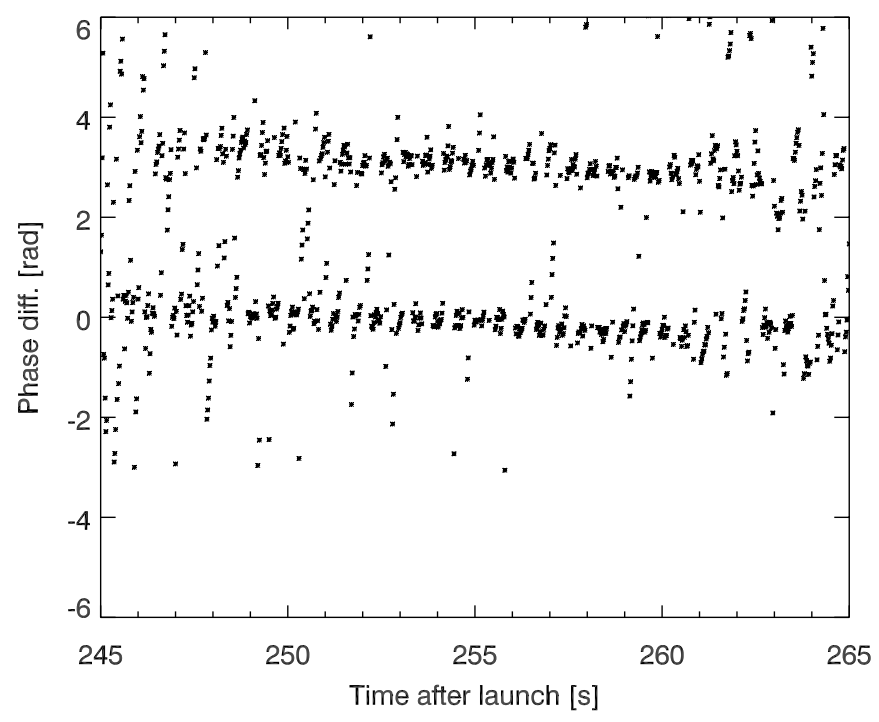

Fig. 11. Relative variation in direction of propagation for downleg conditions.

in terms of the ion-neutral collision frequency $v_{i}$ and the ion cyclotron frequency $\Omega_{c i}$. The directional angle of the steady state ion drift with respect to the $\mathbf{E}_{0} \times \mathbf{B}$-direction is consequently given by $\Psi=\operatorname{ArcTan}\left(v_{i} / \Omega_{c i}\right)$. Using an average value $\sim 200 \mathrm{~s}^{-1}$ for the ion cyclotron frequency we find from Fig. 3 that the ratio $v_{i} / \Omega_{c i}$ varies by approximately from 40 to 5 in the altitude interval $95-105 \mathrm{~km}$, implying a change in $\Psi$ of approximately $89^{\circ}$ to $77^{\circ}$, which is consistent within a factor of 2 with the observed variation in the direction of propagation from Fig. 12. We note here that one reason for uncertainty in estimate originates in the use of an average ion cyclotron frequency, but also the basic ionospheric model implied in Fig. 3 has some uncertainties. Nonetheless, we find it interesting that a simple collisional altitude variation is only marginally sufficient for explaining the observations of variations in directions of wave propagation. Nonlinear wave effects can seemingly be excluded, since the variations are almost the same for upleg and downleg conditions, while the rms-wave amplitudes (as detected by the probe potential differences) vary by almost a factor 2 .

For reference we show in Fig. 13 the altitude variation of the local rms-fluctuation level for the signal $U_{6}(t)$ for upleg and downleg conditions. The result is given in units of $\mathrm{mV}$ for the potential difference between the two front probes, see Fig. 2. Figure 13 illustrates the altitude ranges (for upleg and downleg conditions) with significant wave amplitudes, and serves also to give the conversion between times after launch and the rocket altitudes. As we might expect, the uncertainty on the estimate for the phase velocity, as seen in Fig. 9, becomes large when the intensity of the unstable waves is small, see Fig. 13.

The analysis of this section implicitly assumes that the waves are non-dispersive, at least to a good approximation, 

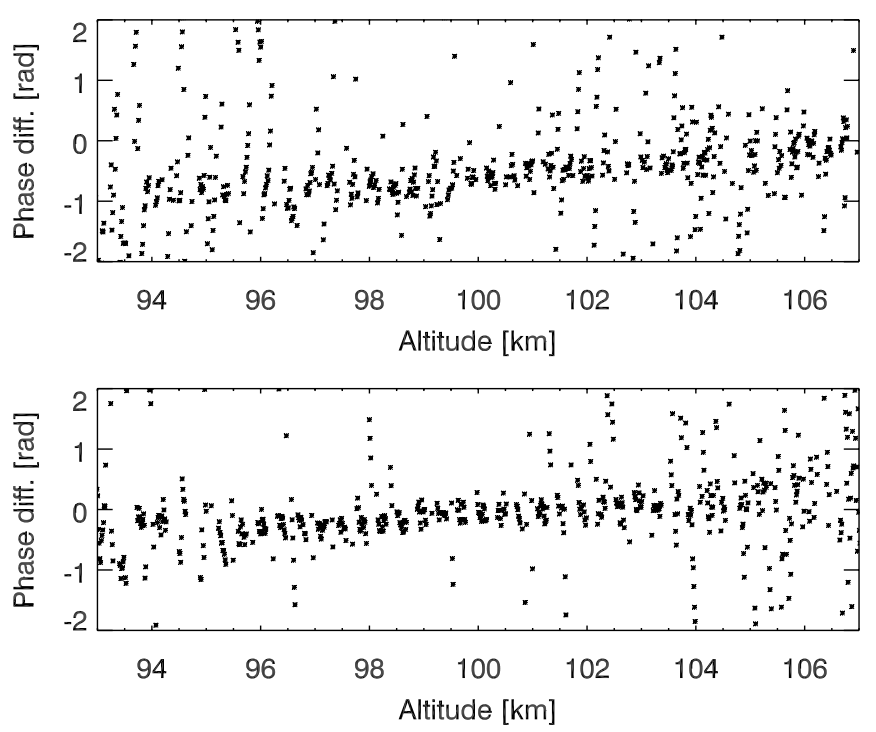

Fig. 12. The relative variation in direction of propagation for upleg (top) and downleg (bottom) conditions, as a function of altitude.

and can therefore be assigned one characteristic propagation velocity. We have tried to test this hypothesis by several methods, for instance by band-pass filtering the data prior to cross correlation (Iranpour et al., 1997) and found some indications of higher frequencies having the largest phase velocities, but the uncertainty on the estimate was significant. A cross-phase analysis (Krane et al., 2000) did not give conclusive results on this question. We find it most safe to argue that the largest amplitudes detected have a constant phase velocity as given by Fig. 9, and leave the phase velocity of the high frequency small amplitude component open, although we expect these phase velocities to be increasing rather than decreasing.

The data-analysis indicates that the ionospheric fluctuations being detected are strongly magnetic field aligned, consistent also with radar observations (Balsley, 1969) and some laboratory studies of these instabilities (Alport et al., 1981). This observation is substantiated best for the downleg part of the flight where the rocket trajectory is close to be magnetic field aligned. The coherence time for the component along the rocket axis is longer than for the one in the transverse direction, indicating $k_{\|} \ll k_{\perp}$, as discussed also by Iranpour et al. (1997) and Krane et al. (2000). The linear stability analysis based on (1)-(2) or similar results also suggests this property of the waves.

To obtain an estimate of the importance of the velocity correction to Fig. 9 as due to the component parallel to the rocket axis, we may consider an altitude of $100 \mathrm{~km}$, corresponding times of flight of $t=114 \mathrm{~s}$ and $t=254 \mathrm{~s}$, for upleg and downleg conditions, respectively. The absolute value of the vertical velocity components are here $660 \mathrm{~ms}^{-1}$. After some calcula-
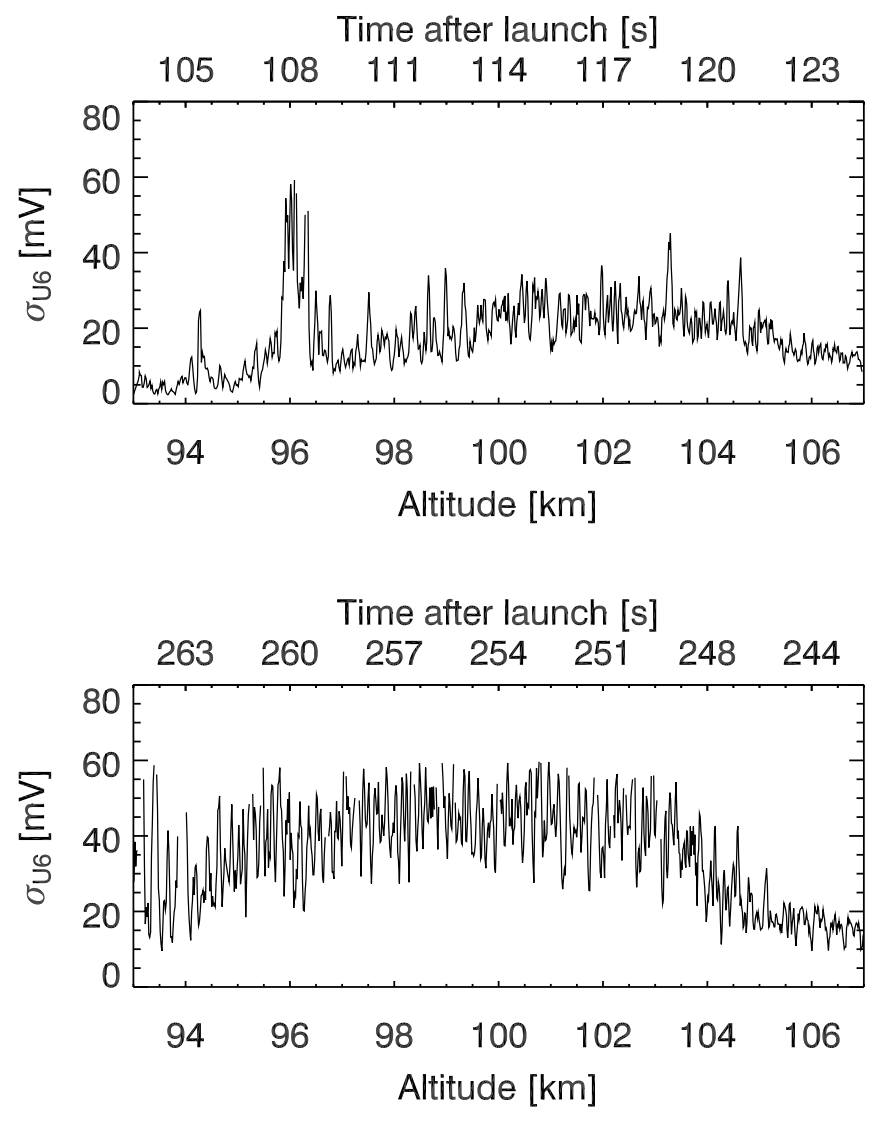

Fig. 13. Altitude variation of the local rms-fluctuation level for the signal $U_{6}(t)$ for upleg (top) and downleg (bottom) conditions. The results are obtained by a moving average over $80 \mathrm{~ms}$.

tions, we find an expression for the time delay between the two probes along the rocket axis to be

$\Delta t=\ell \frac{\widehat{\mathbf{k}} \cdot \widehat{\mathbf{s}}}{u_{p h}-\widehat{\mathbf{k}} \cdot \mathbf{U}}$,

where $\ell=185 \mathrm{~cm}$ is the separation between the two probesets, see also Fig. 2, and $\widehat{\mathbf{s}}$ is the directional unit vector along the rocket axis, having essentially constant direction during the flight. We introduced $\widehat{\mathbf{k}}$ as the unit wave-vector (assuming non-dispersive waves), while $\mathbf{U}$ is the rocket velocity vector and $u_{p h}$ is here the total phase velocity of the waves. From Eq. (3) and the observed time delays in the top frames of Figs. 5, 6, 7 and 8 , we find that since $\Delta t \neq 0$, the waves must have a wave-vector component along the rocket axis, i.e. $\widehat{\mathbf{k}} \widehat{\mathbf{s}} \neq 0$. Figure 9 refers only to the phase velocity component $\perp \widehat{\mathbf{s}}$, but noting that it shows very little variation between upleg and downleg conditions, we anticipate that also the total phase velocity is relatively constant during the flight, apart from the small shift in altitude already mentioned. From Fig. 12 we may expect that the direction of propagation change only a little as well. The differences between the observed time-lags for up-leg and downleg conditions are 
likely to be found mainly in the variation of $\widehat{\mathbf{k}} \cdot \mathbf{U}$, where $U_{v}$ changes sign from upleg to downleg conditions. Allowing for a wave-vector component along the rocket axis, we find that the phase velocities summarized in Fig. 9 are an overestimate, and that the absolute value of $u_{p h}$ can be approximately $10 \%$ lower, although this correction has a significant uncertainty.

We find it interesting to note that the ratios of the rmsvalues $\sigma_{U_{6}}$ for the signal $U_{6}(t)$ for upleg and downleg conditions in Fig. 13 have a typical value of $\sim 1.8$, which is quite close to the ratio $70 / 40$ of the electric fields for those conditions. Although we only have these two values available here, we note that the observation is consistent with the scaling of the power of radar backscatter with the ionospheric drift velocity observed elsewhere (Balsley, 1969; Balsley and Farley, 1973) for a related instability.

\subsection{Spectral analysis}

The data, showing fluctuations in probe-potential differences as well as in relative density, represent a non-stationary dataset, because the rocket is traversing an inhomogeneous ionospheric plasma. The data can be analyzed best by wavelet methods, which better highlight local spectral features (Wernik, 1996; Mallat, 1998). We consider these methods for standard, and do not enter a more detailed description. In Fig. 14 we present samples of local spectra obtained by this method. We find that the observed fluctuations are characterized by two spectral subranges: for low frequencies, below approximately $100 \mathrm{~Hz}$, we note discrete peaks in the spectrum, where these peaks are often harmonically related, i.e. $f_{2} \approx 2 f_{1}$, and sometimes by $f_{1}+f_{2} \approx f_{3}$, where the the bispetral analysis indicates that these relations are phase coherent. These discrete spectral characteristics are transient, and observed often for only a fraction of the rocket spin. For large frequencies, $f>100 \mathrm{~Hz}$, we find a continuous power spectrum, which can be well described by a simple power law, $S(f) \sim f^{-\alpha}$, where the spectral index for potential fluctuations is typically in the range $\alpha_{\phi} \in\{2.5,3\}$, while it is $\alpha_{n} \in\{1,1.5\}$ for the fluctuations in relative density (Schlegel, 1992; Krane et al., 2000), at least in the altitude range most interesting here, $95-105 \mathrm{~km}$. The difference in spectral index for potential and plasma density are, within an experimental uncertainty, consistent with a proportionality between electric field and density (Mikkelsen and Pécseli, 1980; Krane et al., 2000). Since here only time-series are available, we can determine power-law subranges for the frequency spectra only. Since, however, the waves are found to propagate with a relatively high velocity, it is plausible that by use of Taylors hypothesis (Shkarofsky, 1969; Hinze, 1975) for "frozen turbulence" (relevant for a probe propagating rapidly through a spatially varying turbulent velocity field), we might argue for this subrange being characteristic for a component of the wavenumber subrange as well, taking the component along the preferred direction of propagation. The electric circuits

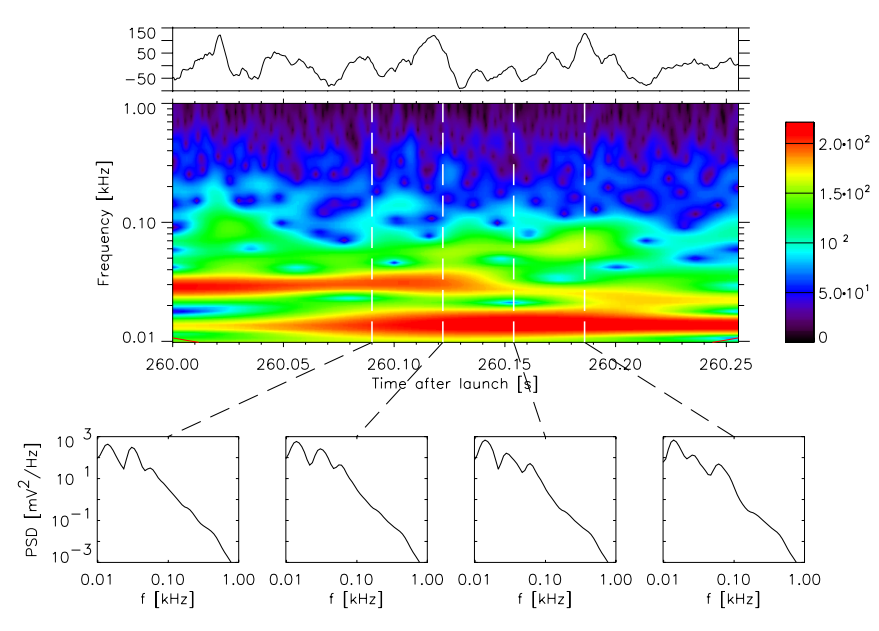

Fig. 14. Samples of spectra obtained by wavelet transform of rocket data, here the $U_{6}$ signal. The times after launch are indicated on the horizontal axis. The data belong to the time-interval analyzed also in Fig. 4. The high frequency part of the signal follows a power-law $f^{-3}$ to good accuracy in this time-interval, in agreement also with results of Krane et al. (2000).

have constant amplification in the relevant frequency range, and will thus not distort the spectra. All frequencies are well below the ion-plasma frequency, so we can assume sheath resistances and coupling coefficients to be constant through the same frequency ranges. The ultimate short wavelength cut-off is likely to be determined by the size of the probes, here $5 \mathrm{~cm}$. We do not expect it to be possible to detect wavelengths shorter than this, and note that with the velocity of propagation found experimentally, this wavelength would correspond to frequencies in excess of $3 \mathrm{kHz}$, which is above the cut-off frequency of the detecting system.

The observed spectral shapes are not too different from the Kolmogorov-Oubokhov law $f^{-5 / 3}$, obtained by use of Taylors hypothesis. Since the neutral gas in the upper parts of the ionospheric D-region (Thrane et al., 1981), and most likely also the lower parts of the E-region, are at times characterized by strong velocity fluctuations, the question naturally arises: to what extent is this neutral turbulence capable of "shaping" the power spectrum of the fluctuations originating from the distributions of plasma charges? We recall here that the ionneutral collision frequency is sufficiently large to let the ion component be transported by a neutral flow by the collisional interactions (Tchen, 1973; Schlegel and Gurevich, 1997). Indeed, if we let the ions be transported by the neutral turbulence as a purely passive contaminant, the analysis can be made relatively simple (Leslie, 1973). More detailed investigations (Gurevich et al., 1997) take into account the different mobilities of electrons and ions. Most of these models refer, however, to regions below $100 \mathrm{~km}$, and it is not evident to what extent these effects are relevant for the interpretation of the present data. 
Table 2. Input data for the "strongly driven" numerical simulations.

\begin{tabular}{lcl}
\hline$B$ & $5.086 \times 10^{-5}$ & magnetic field, Tesla \\
$E_{0 z}$ & 0.00 & dc-electric field in V/m, x comp. \\
$E_{0 x}$ & -0.070 & dc-electric field in V/m, z comp. \\
$-e$ & $-1.6022 \times 10^{-19}$ & electron charge, Coulomb \\
$m_{e}$ & $9.11 \times 10^{-31}$ & electron mass, kg \\
$v_{e n}$ & 28118.4 & electron-neutral coll. frequency, Hz \\
$T_{e}$ & 324.9 & electron temperature, $\mathrm{K}$ \\
$n_{n}$ & $5.05 \times 10^{18}$ & neutral number density, $\mathrm{m}^{-3}$ \\
$T_{n}$ & 216.600 & neutral temperature, $\mathrm{K}$ \\
$M_{i}$ & $5.0 \times 10^{-26}$ & effective ion mass, kg \\
$q_{i}$ & $1.6022 \times 10^{-19}$ & ion charge, Coulomb \\
$n_{i}$ & $5.159 \times 10^{10}$ & number density of ion-species, $\mathrm{m}^{-3}$ \\
$\nu_{i n}$ & 2109.31 & ion-neutral coll. frequency, Hz \\
$T_{i}$ & 216.6 & ion temperature in $\mathrm{K}$ \\
\hline
\end{tabular}

In order to analyze possible couplings between different spectral components, we use a wavelet-based bicoherence analysis (Wernik, 1996; Larsen et al., 2002). This analysis can reveal a possible phase-coherence between different frequency components, which satisfy a resonance condition $f_{3}=f_{1}+f_{2}$. In Fig. 15 we have show local bispectra obtained by wavelet methods for the same time interval as analyzed in Fig. 14. In order to reduce the amount of information in the bicoherence to a more manageable level, we show also the "summed bicoherence" obtained by summing all bicoherence values corresponding to the same $f_{1}+f_{2}$ and dividing by the number of terms entering the sum. The analysis is carried out for a time intervals larger than shown, so "edge effects" are negligible here. We have analyzed a larger part of the rocket data, but the results shown here can be considered representative. The bicoherences on the other hand are strongly intermittent: in bursts it can exceed two standard deviation from the synthetic "null signal" used for reference here. Also this feature agrees with previous results by Larsen et al. (2002), where the same dataset was analyzed by different methods. Also data from one of the Greenland rockets analyzed before (Pécseli et al., 1993) indicated statistically significant bicoherences, but also here of a bursty or localized nature.

In order to provide an estimate for the statistical significance of the results we obtain a synthetic dataset (or "surrogate data"), using standard methods (Wernik, 1996). These data have the property that their amplitude distribution is Gaussian and the power spectrum is identical to the original spectrum, but all phase coherencies have been randomized, and ideally the bicoherencies should be vanishing. In reality, any dataset of final duration will provide a generally non zero bicoherence. Another source of noise in the wavelet- based bicoherency estimate is intrinsic correlation between wavelet coefficients calculated using the continuous wavelet transform, which is not orthogonal. The aim of using surrogate data is to distinguish between significant and noise dominated data. The results obtained from the synthetic data provide in this context a reference level, which has to be exceeded for the results found in the original data to be significant.

\section{Numerical simulations}

The numerical simulations were conducted in two spatial dimensions in the plane perpendicular to the imposed magnetic field, using a Particle-in-Cell (PIC) code for the ion component (Oppenheim et al., 1995; Oppenheim and Otani, 1996). The electrons are, on the other hand, described by a fluid model. For the results shown in the following, the electron inertia is ignored. We have conducted smaller box simulations with the same parameters as the simulations shown here, but with finite electron inertia, and found no substantial difference in the resulting evolution or spectral characteristics for the present parameters. First we solve the momentum equation for $\mathbf{u}$ by omitting the derivative terms

$0=-\frac{e}{m}\left(\mathbf{E}+\mathbf{u} \times \mathbf{B}_{0}\right)-\frac{\nabla P}{m n_{e}}-v_{e n} \mathbf{u}$.

The electron temperature is assumed to vary adiabatically with respect to the initial temperature, giving $P=P_{0}\left(n_{e} / n_{0}\right)^{\gamma}$, with $\gamma=C_{P} / C_{V}$. Assumptions of isothermal electron dynamics will rely on a finite wavenumber component along magnetic field lines (Pécseli et al., 1989).

Next we solve for the electron density from the full continuity equation

$\frac{\partial}{\partial t} n_{e}=-\nabla \cdot\left(n_{e} \mathbf{u}\right)$.

The ions are treated as collisional and unmagnetized with an effective ion mass and collision frequency listed in Table 1. For these particular simulations the ion magnetization ratio is $\Omega_{i} / \nu_{i n} \approx 0.08$, and we have found no difference in simulation results for ratios below .1, and very little difference until about $115 \mathrm{~km}$ in altitude. Details of the simulation code are given by Oppenheim et al. (2003).

Poisson's equation is retained, so that we need not assume the wave dynamics to be quasi-neutral. Typical parameters for the simulations such as electron-neutral collision frequencies etc., are given in Table 2. Effects of ionization and recombinations are ignored. The ions are represented by an effective ion mass, as indicated in Table 2. Metallic ions can have significant effects (Schlegel, 1985), but these are ignored here. The simulation are carried out on a $512 \times 512$ periodic mesh, using a spectral solution to Poisson's equation. Inspection of the results shows that the simulations evolve in the following manner. First, the development of short 

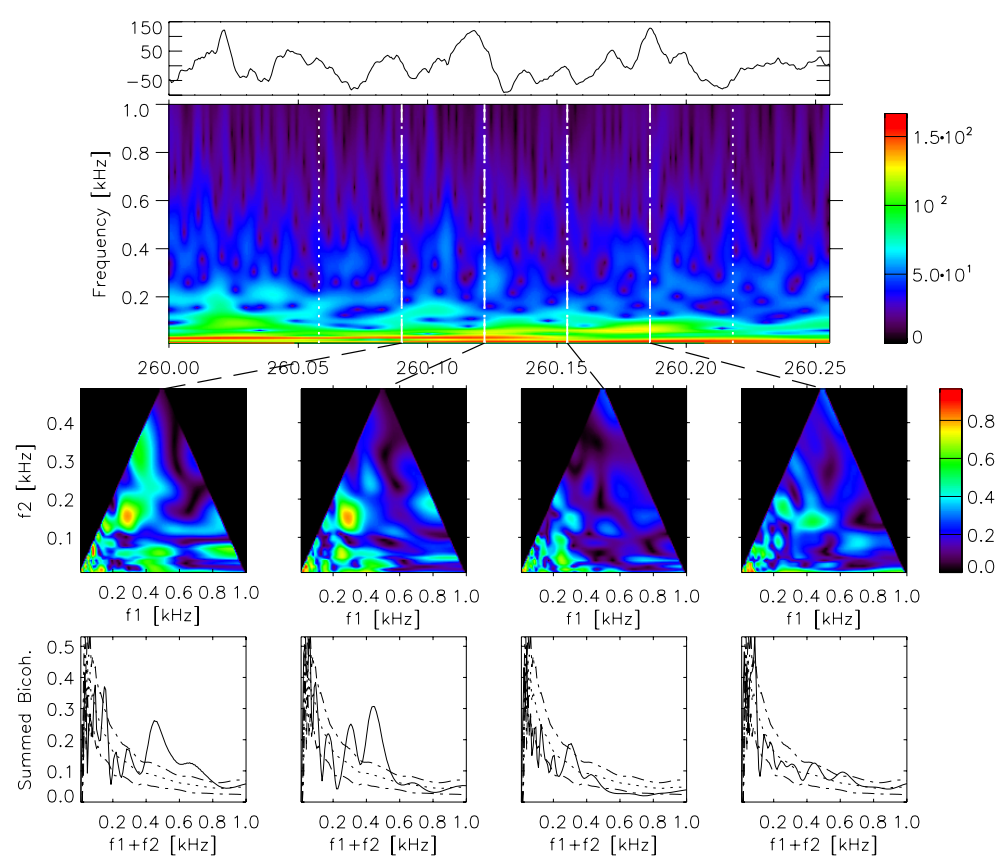

Fig. 15. Samples of bispectra obtained by wavelet transform of rocket data, here the $U_{6}$ signal. The times after launch are indicated on the horizontal axis, see also Fig. 14. The dotted lines in the lowest row give the mean summed bicoherence calculated for 20 surrogate data sets, while dot-dashed lines represent the mean summed bicoherence plus and minus its standard deviation.

scale waves, in agreement with the linear dispersion relation, where the maximum growth rate is for small scales, i.e. large wave-numbers. Later on, the instability saturates with large scale structure, with smaller scale secondary waves superimposed. As mentioned, the results (1)-(2) have limited validity, and refer to one specific plasma model. As an aid for the discussion we show in Fig. 16 examples for linear wave dispersion relations, obtained by three different models, as indicated by labels. The figure shows the solution to the "kinetic" dispersion relation, where the ion density is solved using Vlasov's equation. A dotted line for the real part of the frequency gives the ion sound speed for comparison. The solution with the label "Fluid approx" originates from the standard simplified model suggested by Fejer et al. (1984), while the "full fluid" represents the solution of the full quadratic equation from that same paper without simplification. Since the analysis of kinetic and fluid models are different, we do not attribute any significance to the slight difference between the growth rates of the fluid versus the kinetic models for the long-wavelength limit in Fig. 16. Since we obtain the dispersion relations numerically, we do not need to impose the restriction $\omega_{i} \ll \omega_{r}$, as in Eqs. (1)-(2). A previous analysis by numerical solution of model equations including anisotropic electron thermal conductivities, based on arguments given by Pécseli et al. (1989) was presented by Iranpour et al. (1997). Those results deviate only slightly from those shown here, and the details of the electron thermal conductivity model are most important near threshold electric fields. The results of Fig. 16 are based on the standard local model, while non-
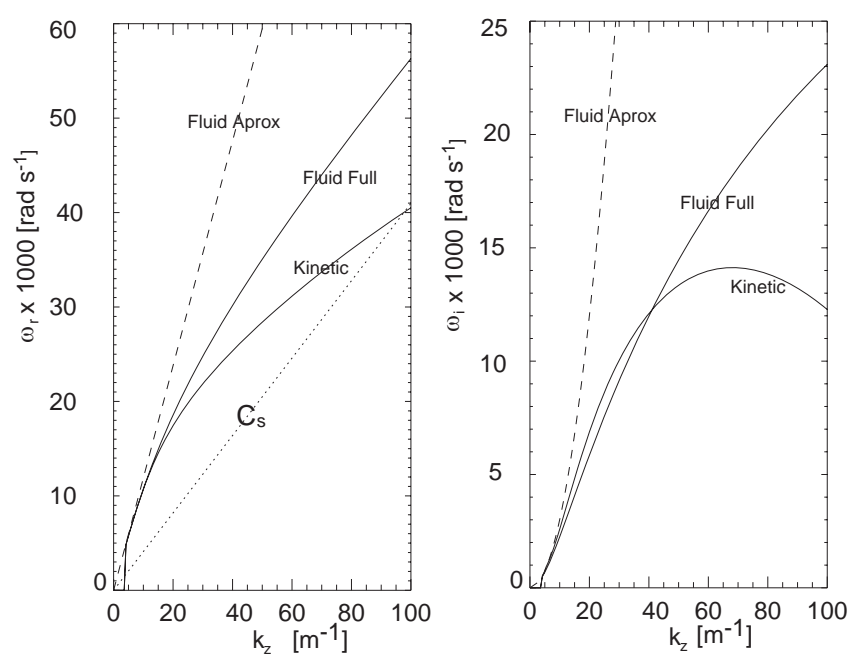

Fig. 16. Numerically obtained dispersion relations, for plasma parameters corresponding to those relevant for the ROSE4 dataset, i.e. altitudes of $105 \mathrm{~km}$ and d.c. electric fields of $70 \mathrm{mV} / \mathrm{m}$. Real and imaginary parts (to the left and right, respectively) of the frequency are shown as a function of wavenumbers in the $\mathbf{E}_{0} \times \mathbf{B}$-direction.

local models have to be applied for strongly inhomogeneous conditions (St.-Maurice, 1985).

In Figs. 17 and 18 we show selected results from the simulations for three different times, chosen so that the first one is in the linear growth period, the second column is just before 

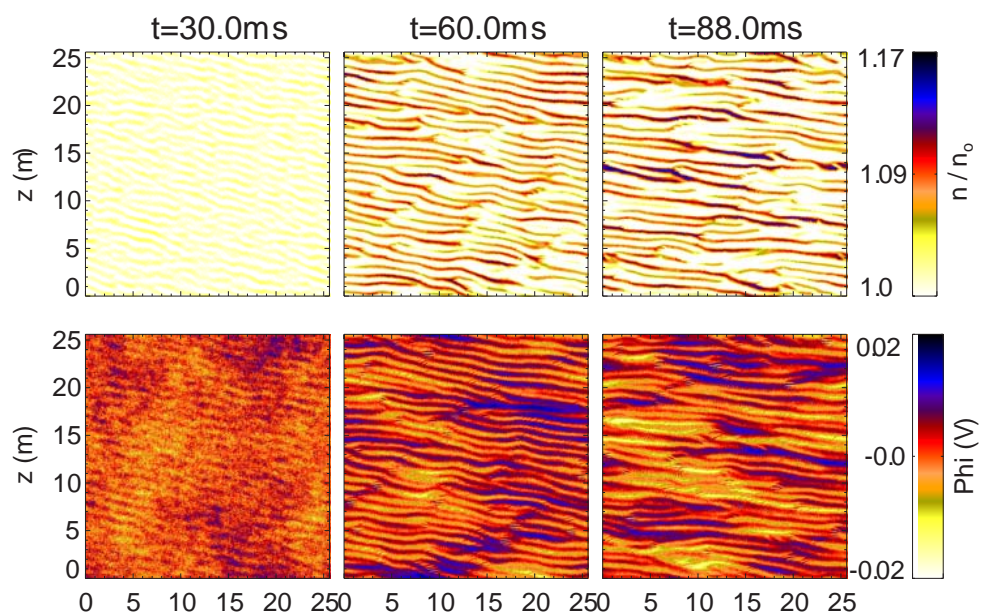

(m)

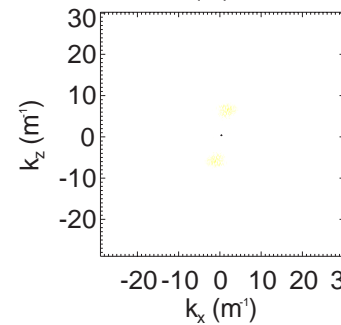

(m)
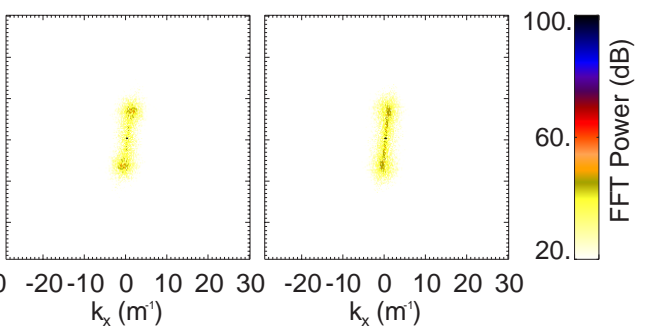
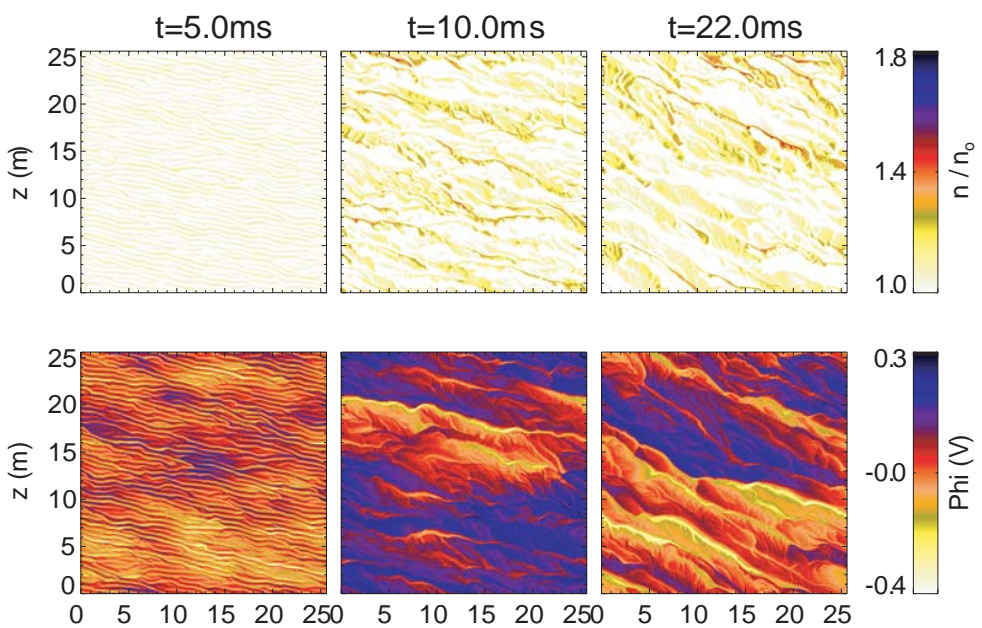

(m)

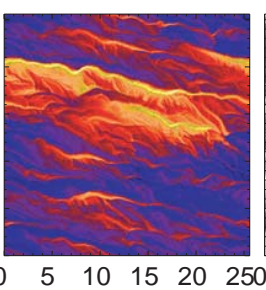

(m)
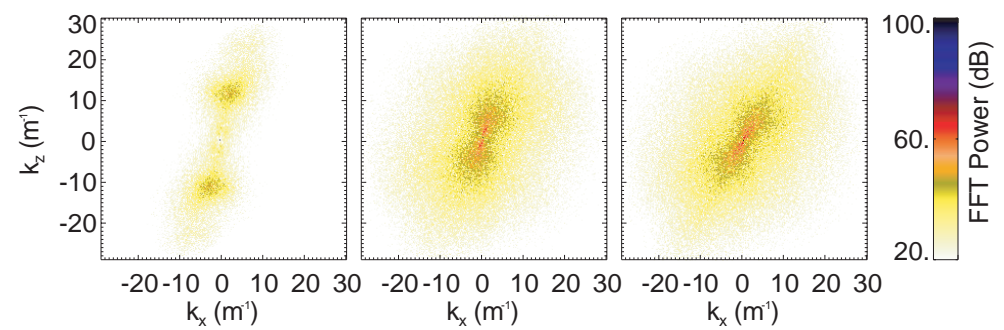

Fig. 17. Summary plots illustrating the results from the numerical simulations for three times. The top panels shows electron density, the middle frames the electrostatic potential, and the bottom frames illustrate the wavenumber spectra for the potential. The magnetic field is perpendicular into the plane of the paper, and the $\mathbf{E}_{0} \times \mathbf{B}$-drift is in the vertical direction, with $\mathbf{E}_{0}=40 \mathrm{mV} / \mathrm{m}$ being in the positive $\mathrm{x}$-direction.

Fig. 18. Summary plots illustrating the results from the numerical simulations for three times. The top panels show electron density, the middle frames the electrostatic potential, and the bottom frames illustrate the wavenumber spectra for the potential. The magnetic field is perpendicular into the plane of the paper, and the $\mathbf{E}_{0} \times \mathbf{B}$-drift is in the vertical direction, here with $\mathbf{E}_{0}=70 \mathrm{mV} / \mathrm{m}$ again in the positive $x$-direction. See Fig. 17 for comparison. 

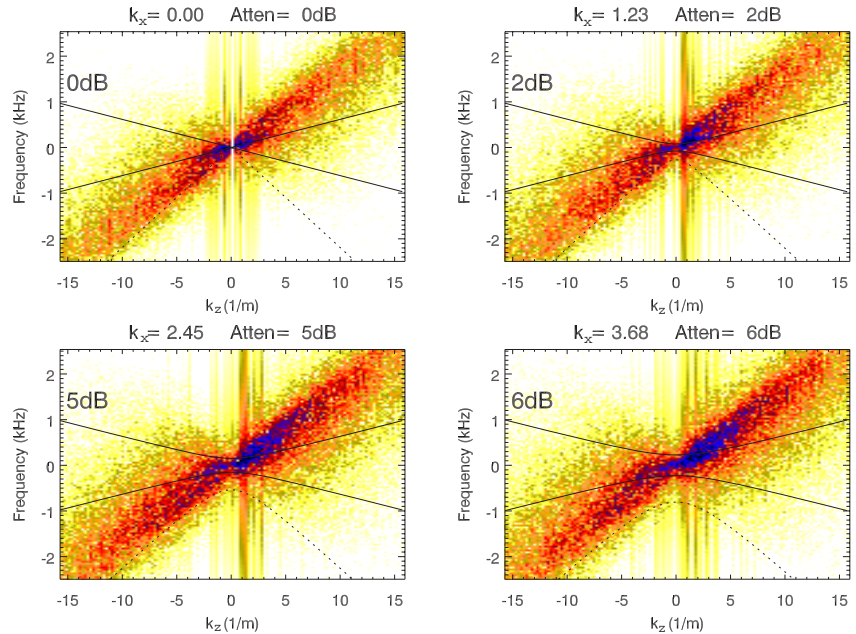

Fig. 19. Plots of the dispersion relations from the plasma simulation shown in in Fig. 18, with $\mathbf{k}_{z} \| \mathbf{E} \times \mathbf{B}$. The slope of the dashed line gives the $\mathbf{E} \times \mathbf{B} / B^{2}$-velocity, while full lines give the sound speed for the given conditions. The dominant direction of propagation is at an angle to the $\mathbf{E}_{0} \times \mathbf{B}$-direction and to improve the presentation the color scale has been amplified accordingly, as indicated.

saturation, while the last column represents a fully developed saturated stage. Times are in units of ms. The first case, Fig. 17, corresponds approximately to our upleg conditions for the ROSE4 rocket, the other one, in Fig. 18, is a strongly driven case, corresponding rather closely to the down-leg conditions for the ROSE4 rocket. We note a significant difference in growth rate of the instabilities for the two conditions. The weakly driven case, Fig. 17, runs for a time interval being four times that of the strongly driven case, and saturates at a less turbulent state then the strongly driven case shown in Fig. 18.

Already in the linear growth phase, we note a deviation between the dominant direction of wave propagation and the $\mathbf{E}_{0} \times \mathbf{B}$-drift direction, see also Fig. 19. This can be due to ion thermal effects (Oppenheim and Dimant, 2004), or the Pedersen ion drift in the direction along $\mathbf{E}_{0}$. This effect is usually ignored in analytical models, but needs evidently to be retained. As the instability saturates, this deviation of propagation-direction from the vertical becomes more pronounced for the strongly driven case (Oppenheim and Dimant, 2004; Dimant and Oppenheim, 2004). Also, we find the general feature of the results to be a large scale structure (basically filling the simulation box) with superimposed smaller scales, which is particularly conspicuous for the strongly driven case, Fig. 18, but noticeable also on enlarged versions of Fig. 17. The numerical resolution is very fine $(d x=d y=5 \mathrm{~cm})$, and by inspection one can find very fine details by enlarging the figure, see for instance Fig. 20 . In particular, the developments of the secondary instabilities (Sudan, 1983) can be clearly seen. We note that such sec-

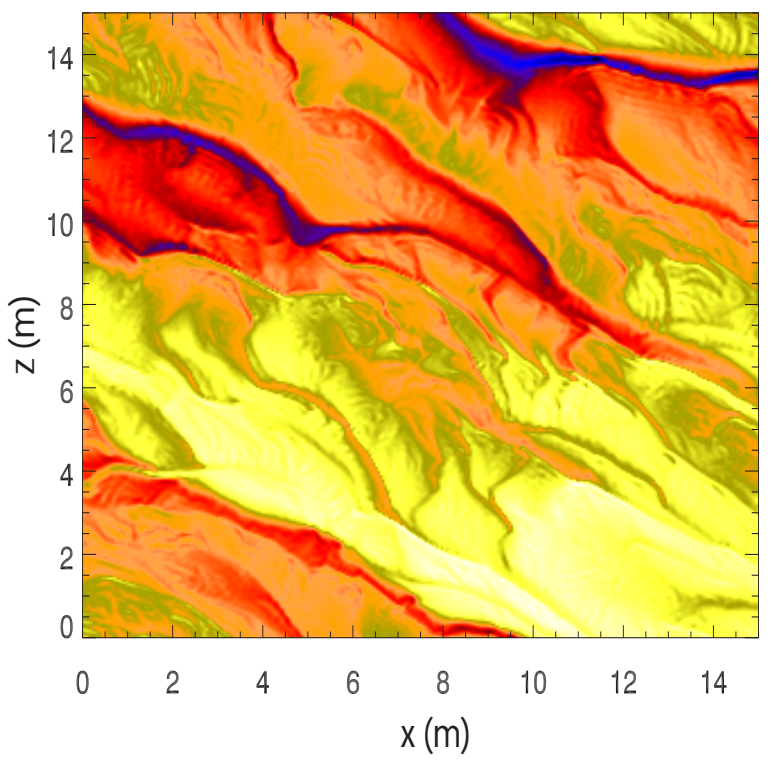

Fig. 20. Enlarged part of the potential variation obtained at the saturated stage of the instability in the strongly driven case, see also Fig. 18. The color code is the same as in Fig. 18. The fine scale secondary instabilities can be recognized as "feather-like" structures, see for instance around $(x, z)=(6,6)$.

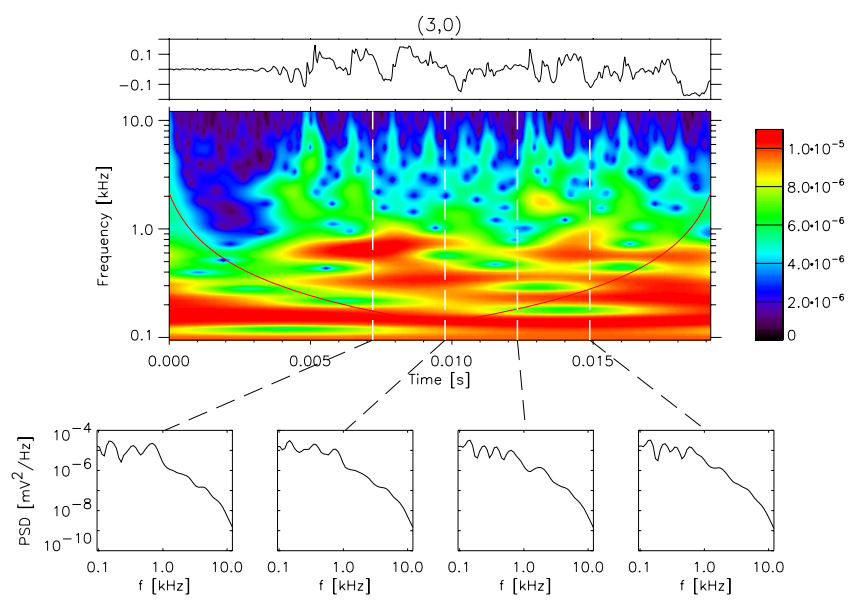

Fig. 21. Spectra for potential fluctuations, obtained by wavelettransform of simulation results at a fixed position, as function of time.

ondary instabilities can be found also for other instabilities (Hallatschek and Diamond, 2003).

In Fig. 19 we present results for the "effective" dispersion relation obtained by Fourier transforming $\phi$ as presented in Fig. 18, in the the spatial and temporal dimensions. We note that the wavelengths here are much larger than what can be determined by analyzing rocket data and even laboratory experimental data, i.e. the wavenumbers are given in physical units with $k=15 \mathrm{~m}^{-1}$ corresponding to a wavelengths as short as $\lambda=0.4 \mathrm{~m}$. The rocket observation correspond to 


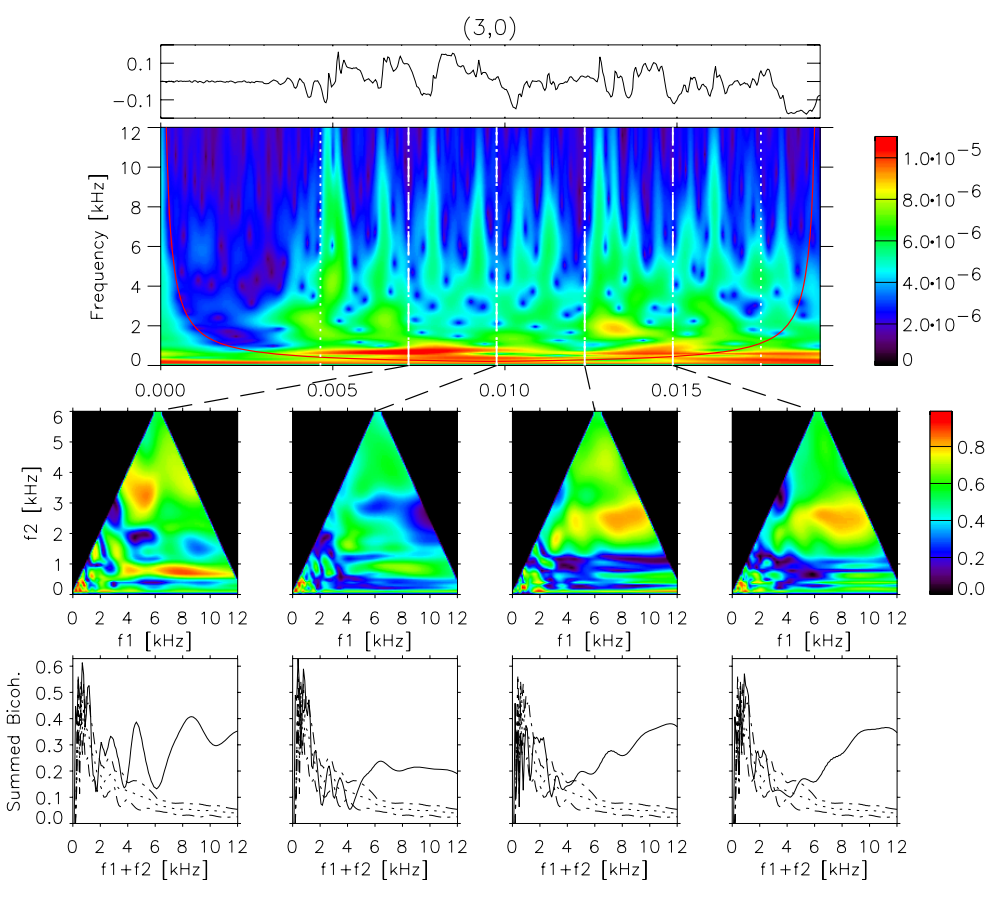

Fig. 22. Bicoherence for potential fluctuations, obtained by wavelet-transform of simulation results at a fixed position, as function of time, see Fig. 21. The dotted and dashed lines in the figures for summed bicoherence, are defined just as in Fig. 15. the small dark area less then $k \approx 4 \mathrm{~m}^{-1}$ in Fig. 19. The first frame with $k_{y}=0$ is symmetric with respect to the origin, but this symmetry is broken is subsequent frames by choosing $k_{y}>0$ there. As a guide for the eye, we have inserted lines for the sound speed, $C_{s}$, and the magnitude of the $\mathbf{E} \times \mathbf{B} / B^{2}$-velocity. (For $k_{y}>0$ these lines are cuts in a cone, and therefore appear as hyperbola.) For the smallest wavenumbers we find the phase velocity to be close to $C_{s}$, to increase for shorter wavelengths. The phase velocity is below the $\mathbf{E} \times \mathbf{B} / B^{2}$-velocity in all cases, but approaches this value for the shortest wavelengths. These results can not explain the exceptionally low subsonic phase velocities found by the analysis of the rocket data, but we find it interesting that they to some extent resolve a paradox concerning some laboratory experiments.

Analyzing waves spontaneously excited under conditions where the Farley-Buneman instability was operative, Mikkelsen and Pécseli (1980) found that the phase velocity increased for increasing frequencies, ultimately to approach the $\mathbf{E} \times \mathbf{B} / B^{2}$-velocity. In their experiment the phase velocity was supersonic for all cases, as supported also by other related experimental observations (D'Angelo et al., 1974; John and Saxena, 1975; Alport et al., 1981). Since these experiments were all carried out in a rotating cylindrical plasma column, it is plausible that the longest azimuthal wavelength $\sim 2 \pi R$, with $R$ being the radius of the plasma column, was too small to reach the region of sonic or subsonic phase velocities. On the other hand, by analyzing rocket data, evidently the emphasis will be on the largest amplitude long wavelength part of the spectrum and there are no similar limits to accessible phase velocities.

\section{Data analysis}

The analysis of the simulated data is carried out in two steps. First we take the simplest and most natural approach, by selecting a typical reference point in the middle of the computational domain, and analyze the spectral characteristics of the signal. We are aware though that this is not directly comparable to what is obtained by the rocket: in this case we have available, as discussed before, the potential difference between two separate probes. The difference between the two cases can be explained by associating a spatial filtering with the difference signal, which is explained best for a spatial Fourier transform of the potential variation. In this representation, the differencing corresponds to a multiplication of the power spectrum by a filter-function $\sin ^{2}\left(\frac{1}{2} \mathbf{k} \cdot \mathbf{d}\right) /\left(\frac{1}{2} \mathbf{k} \cdot \mathbf{d}\right)^{2}$ for spatially homogeneous, but not necessarily isotropic conditions, where $\mathbf{d}$ is a vector pointing in the direction of the vector connecting the two probes, having the length given by the probe separation (Kelley and Mozer, 1973; Pfaff et al., 1984; Krane et al., 2000).

\subsection{One point potential analysis}

We have analyzed the simulation results for comparison with the rocket data. In Fig. 21 we show results for the power spectra for the potential fluctuations obtained from the data 
in a fixed position as function of time. In the top part of the figure we have the raw signal from that spatial position, and note the increase in wave amplitude in the initial linear phase of the instability, until the fluctuations saturate in an irregular oscillation with a mixture of wave periods, where the smallest frequencies seem to have the largest amplitudes. Below the raw data we show, on a logarithmic scale, the wavelet power for the signal. At four selected times after wave saturation, we show local spectra, taking care to integrate the power spectrum over the "cone of influence" for the appropriate mother wavelet, here the Morlet-Grossmann wavelet. It is interesting to note the close agreement with the spectra obtained from the rocket, as summarized by Krane et al. (2000). We thus note a "spiky" structure for low frequencies and a continuous power-law spectrum at higher frequencies, in agreement also with the laboratory experiments by Mikkelsen and Pécseli (1980). The agreement is also quantitative, by giving peaks in the spectra in the range up to $\approx 200 \mathrm{~Hz}$. In the power law part of the spectrum we find the exponent to be close to the value $\sim 2.5$ observed by Krane et al. (2000) and Mikkelsen and Pécseli (1980). The nature of the fluctuations is strongly intermittent, as best seen by the color coded spectrum, in particular in the high frequency parts.

In Fig. 22 we show results for the bicoherence, again obtained by wavelet-techniques. In order to reduce the amount of information in the bicoherence to a more manageable level, we show also here, at the same times as the spectra were obtained in Fig. 21, the "summed bicoherence" obtained by summing all bicoherence values corresponding to the same $f_{1}+f_{2}$ and dividing by the number of terms entering the sum. The bicoherence was calculated over 128 points centered at the chosen time instant and for 128 frequencies. This method of data-reduction is standard, and allows presentation of results as a function of one variable only. We note significant peaks in the bicoherence, but taking into account the short time series used for obtaining these results, we have ample reasons for caution. It is thus well known (Bendat, 1958; Pécseli and Trulsen, 1993; Pécseli, 2000) that analysis of short data sequences often give spurious results. Consequently, also in this case we produced a synthetic dataset, in order to have a reference when estimating the statistical significance of our estimates (Wernik, 1996). The synthetic data were produced to have no phase coherencies, and were analyzed the same way as the original data. The results are shown with dotted lines for the average of 20 Gaussian sets obtained this way, and \pm 1 standard deviation is given by dot-dashed lines in the summed bicoherence figures in Fig. 22. It is readily seen that the large values of the observed bicoherencies at small frequencies are completely fictitious, as could be expected, since the width of the mother wavelet is here comparable to the integration interval. For larger frequencies we note, however, that the observed bicoherence is noticeably above the average + one standard deviation, and here the bicoherence is statistically significant.

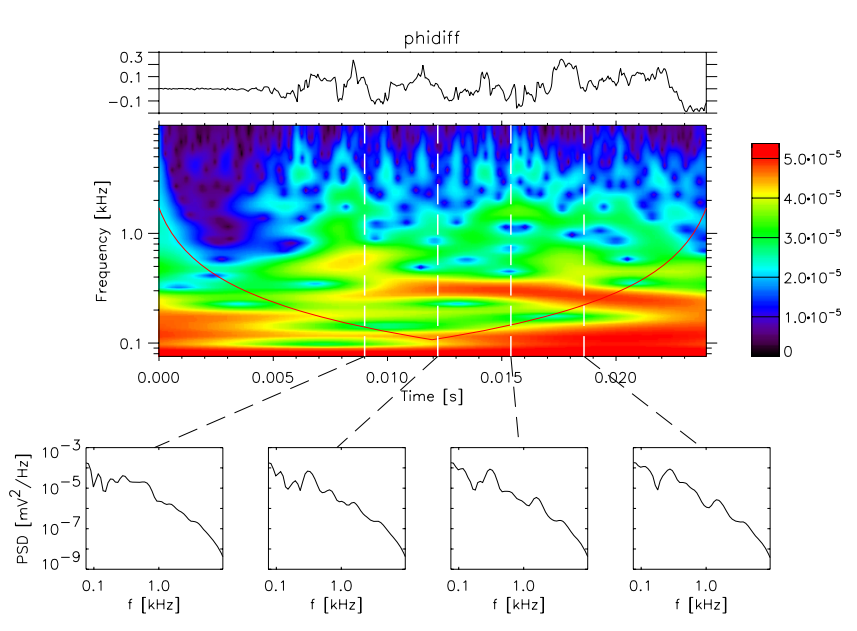

Fig. 23. Wavelet spectrum of signal obtained by the potential difference between two points, separated by $3 \mathrm{~m}$ in the $\mathbf{E}_{0} \times \mathbf{B}$-direction.

The physical origin of a finite bicoherence in the spectrum can of course not be explained by these observations alone. Considering the "spotted" nature of the enhanced regions in the bicoherence plots, we find it reasonable to argue that the observations indicate phase-coherent couplings of enhanced spectral components, but note also the transient nature of these interactions.

One of the problems associated with the observed structures in the low frequency part of the spectra was pointed out by Krane et al. (2000): the dispersion relation has no local maxima at low frequencies or long wavelengths, see Fig. 16. The most obvious explanation advocated then was the presence of striations in the background plasma, which might somehow give rise to some "selection laws" for the most unstable long wavelengths. Indeed, indirect evidence could be argued for the presence of such striations. We also find low frequency structures in the numerical simulations, despite the homogeneity of the spatial conditions assumed in the simulations. The initial growth phase has no predominant large scales present. The low frequency "spiky" part of the spectrum thus seems to be natural for the saturated stage of the Farley-Buneman instability. We might argue that seemingly, if stationary density striations are present in the ionosphere, then they do not have any pronounced influence on the wave characteristics.

\subsection{Two point potential difference analysis}

In Fig. 23 we show a wavelet-based power spectral analysis of the potential difference between two positions with $3 \mathrm{~m}$ separation along the direction of the $\mathbf{E}_{0} \times \mathbf{B}$-direction, obtained from the simulations. One of the positions is the one giving the signal in Figs. 21 and 22. The separation chosen represents closely the probe separation on the rocket. The spectra are somewhat distorted as compared to the original one point data in Fig. 21, but the local power spectra are 


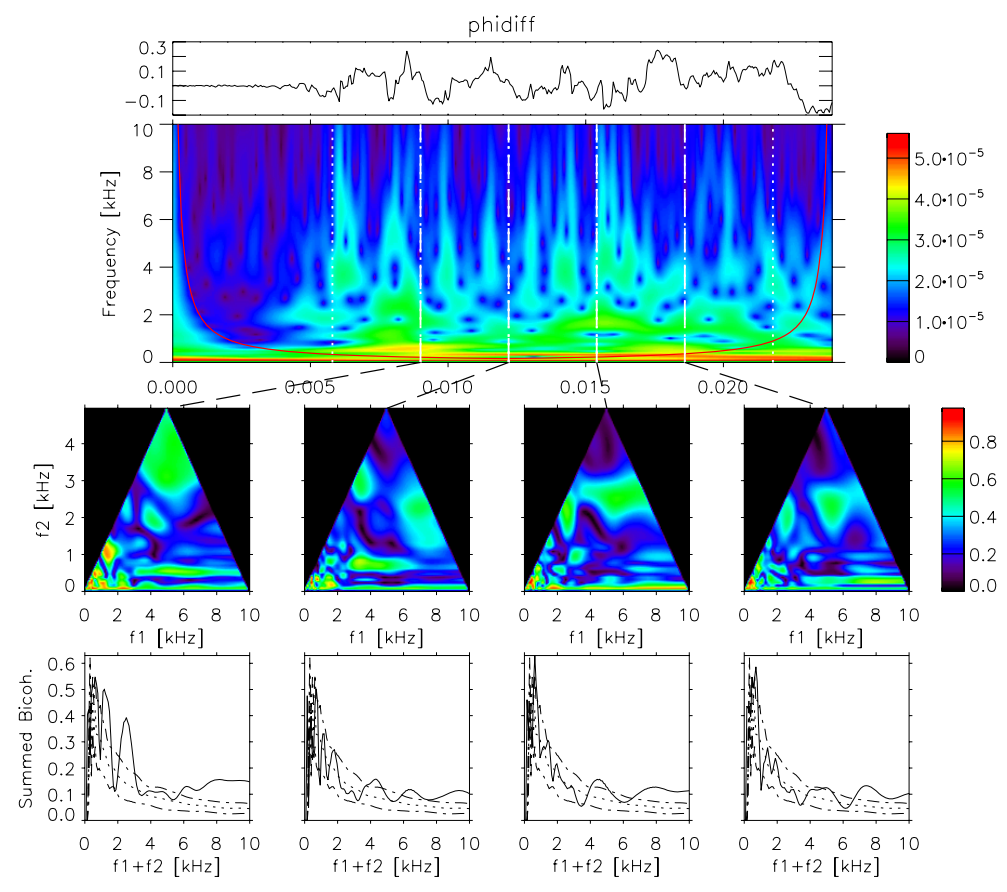

Fig. 24. Bicoherence spectrum for the dataset analyzed in Fig. 23. still recognizable. The spectral indexes are approximately the same, although we expect that it is necessary to take averages over longer samples to get a clear result. The high frequency continuous part seems to extend to somewhat lower frequencies here, and the low frequency "spikes" are not as pronounced as in Fig. 21.

Figure 24 shows the bicoherence for the probe difference signal obtained as in Fig. 21. By comparison with the results from the original data, illustrated in Fig. 21, we find that the bicoherences are significantly distorted, in particular at the high frequency parts. If we emphasize the low frequency part of the bicoherence we find results like those shown in Fig. 25, which incidentally resembles the results from the rocket data.

The effective filtering mentioned before applies to the instantaneous potential field. For wavelengths much shorter than the probe separation, it might be argued that one probe here simply acts as a reference for the other one (Krane et al., 2000). The difference between the results from the one-point data analysis and the one based on the probe-difference signal may appear surprisingly large, but it should be emphasized that the spatial filtering is followed by a time-averaging implied by the wavelet analysis. The consequences of these two combined steps is not intuitively obvious.

The essential observation based on the results from this subsection is that the probe difference data represents a rather distorted version of the potential variations in the plasma. In particular the bicoherences are severely affected by the effective filtering due to the differencing. If we find signs of bicoherent couplings in the spectrum, these might very well be much stronger in the fluctuating fields of the ionospheric plasma. Although our analysis refers explicitly to instrumented rockets, these conclusions have implications for satellites as well, when waves are sampled by potential differences between two separated probes.

\section{Conclusions}

We have analyzed irregularities in the ionospheric E-region, as detected by instrumented rockets from the ROSE experiment. Figures 5, 6, 7, 8, 10 and 11 contain most of the relevant information, but not in the preferable form since the variable is the time of flight. In Figs. 9 and 12 we present the most important information deduced from the rocket data for varying altitudes. The analysis was supplemented by numerical simulations.

A few basic observations can be made right-away: the difference in dc electric fields for upleg and downleg conditions (40 and $70 \mathrm{mV} \mathrm{m}^{-1}$ ) is reflected in the differences in rms wave activities observed, see Fig. 13. We note an altitude shift, consistent with the altitude shift of the enhanced fluctuation level. Apart from this, we do not find any difference (that is: not within the statistical uncertainty) of the observed phase velocities, implying that at least within the present parameter range, there is no dependence of the phase velocities on the corresponding wave amplitudes. Similar comments apply to the directional changes of the wave propagation with altitudes. The observed changes can be explained in part by the variations in Hall and Pedersen ion velocities due to the altitude variations of the ion-neutral collisions, see Fig. 3. 


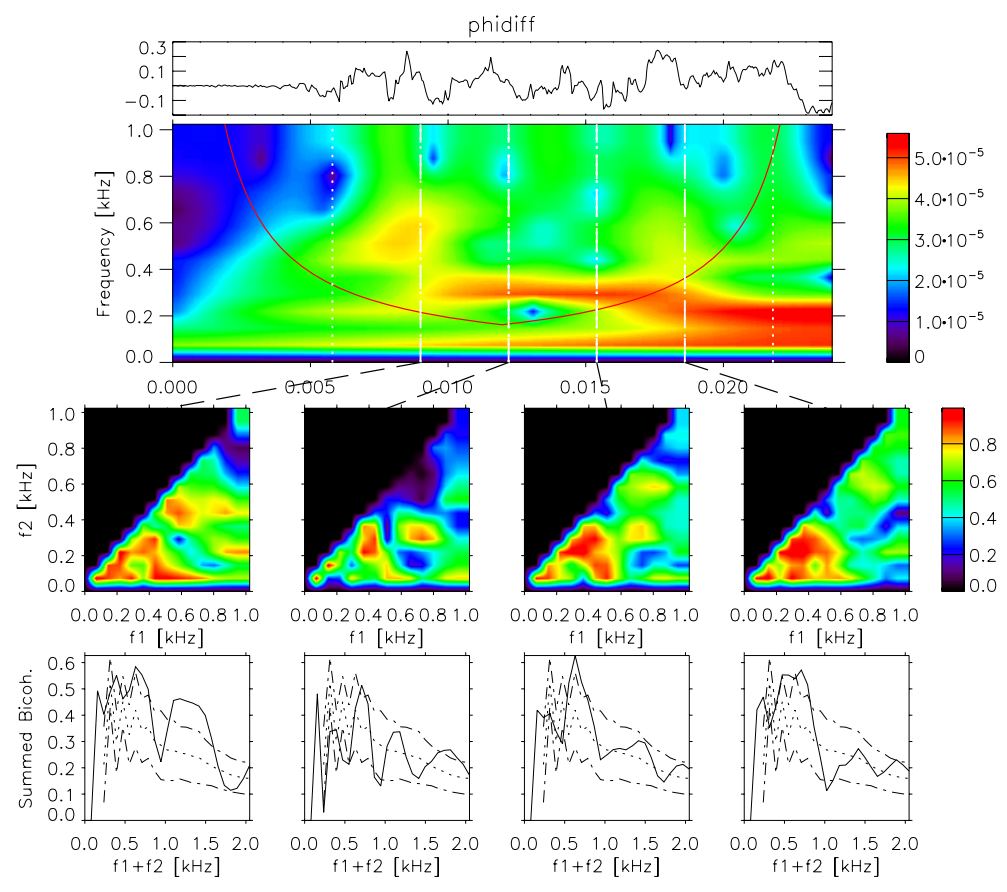

Fig. 25. Enlarged version of the low frequency part of Fig. 24, see also Fig. 23. The present figure shows some indications of a significant bicoherency in the first frame only, otherwise the signal is within the uncertainty of the "null" or "synthetic" signal.
A slight difference between the directional change for the upleg and downleg conditions can be argued, but we find it to be within the experimental uncertainty. One restriction on these conclusions is of course that they refer to the dominant wave amplitudes only. For large frequencies, the spectral energy decreases, and estimates of, for instance, ionospheric phase velocities become uncertain.

Based on the rocket data alone, we can not make definite statements concerning steady state large scale density gradients in the ionospheric plasma. In case such gradients have components along the rocket trajectory, they would have been observed by the density probes on the rocket. However, apart from the vertical density gradient usually associated with the E-region (Rose et al., 1992), we see no signs of such a density gradient. One possibility is that the rocket flight was along equi-density contours of a steady state density gradient, which would not be detected. In that case, however, the direction of the gradient would be along the electrojet current direction, and it is difficult to see how such a steady state gradient could be maintained. We argue that it is unlikely that any steady state density gradients with a component perpendicular to the magnetic field are present during the ROSE4 flight, except for the natural vertical gradient. Consequently, our numerical simulations are ignoring such large scale density gradients.

The simulations gave results which in many respects agree with the rocket data, qualitatively as well as quantitatively. In making comparisons with the rocket observations we found it important to mimic the rocket measurements in detail by taking the potential difference between two points separated by the rocket boom length. The best agreement was found for the strongly driven (down-leg) case. The fluctuation levels are approximately the same, and the velocities of wave propagation are unidirectional and have comparable phase velocities at long wavelengths. The simulated spectra also have a low frequency, long wave-length, "spiky" component, followed by a continuous spectral range well approximated by a power law with index close to -3 . The simulations as well as the rocket data are characterized by strongly intermittent bicoherencies. Important differences are found in an apparent "shift" in frequency range, i.e. in the simulations the spiky subrange continues up to approximately $0.5-1 \mathrm{kHz}$. In the simulations, we also have indications for a change in direction of wave propagations induced by nonlinear wavephenomena. This effect does not have any counterpart in the rocket observations, where the observed change in direction is the same for upleg and downleg conditions in spite of change in dc-electric fields from $40 \mathrm{mV} / \mathrm{m}$ to $70 \mathrm{mV} / \mathrm{m}$.

The numerical simulations indicate that the smallest scales in the wave-fields are driven by secondary instabilities, and that the characteristics of this part of the spectrum changes from one side of the large amplitude wave-crests to the other. This can explain the strong intermittency of the bicoherence couplings observed also in the analysis of rocket data, which agree also with other observations (Pécseli et al., 1993; Larsen et al., 2002). We emphasize again the significant difference between the one-point statistics of the numerically simulated potential variations and the two-point potential difference signal: the latter, which closely mimics the potential measurements by the rocket instruments, gave a close 
to excellent agreement with the analysis of the in-situ data. These conclusions evidently refer to probe configurations as shown in Fig. 2. Significantly different configurations have to be analyzed separately.

We find that the close agreement between the observed spectra and those from the simulations allow us to draw some nontrivial conclusions. First, we recall that the simulations are restricted to the two spatial dimensions perpendicular to B. It can thus be argued that the B-parallel wave dynamics is of minor importance for the development for the spectrum for the strongly driven case. Since the neutral atmosphere is taken to be at rest in the simulations, and the results are in good agreement with observations, it seems that we might safely conclude that the observed spectra are the results of plasma nonlinearities, and not a consequence of mixing by turbulence in the neutral background atmosphere. Neutral turbulence is, in our opinion, unlikely to have a major role for the details of the electrojet fluctuations for conditions corresponding to the ROSE flights.

The most significant discrepancies between the in-situ data and the numerical simulations are, in our opinion, the difference in directional variation of the wave-propagation. The simulations indicate a nonlinear effect, while the observations unambiguously speak against such effects. Also we find a nontrivial difference in the speed of wave propagation, but note here that a uniform neutral wind might imply that the actual velocity with respect to a neutral atmosphere is different from the one found here. (We also note that the Greenland rocket launched in 1976 under conditions very similar to the ROSE4-flight gave phase velocities very close to, or slightly above, the sound speed, as described by Pécseli et al., 1989). These features can justify that our numerical studies are extended by a fully three dimensional numerical simulation, to ensure that they are not artifacts induced by the limitations of a two dimensional model as the one used here. On the other hand, we find it safe to argue that the basic properties of the turbulent low frequency wave-field are well accounted for by the present simulations in two spatial dimensions.

\section{Appendix A}

\section{Electron temperature in the electrojet}

As stated before, we are not able to give any accurate experimentally obtained value for the electron temperature. This parameter is of some relevance for the interpretation of our results, and there has been discussions on the possibility for the Farley-Buneman instability to enhance this temperature. One possible scenario for nonlinear saturation of the instability is actually based on the assumption that $T_{e}$ can be enhanced sufficiently to make the sound speed close to the $\mathbf{E} \times \mathbf{B}$-drift velocity of the electrons, thereby reducing the linear growth rate of the instability, and ultimately stabilize the waves. Unfortunately, it seems that these arguments are based on over-idealized assumptions for infinite plasmas, and we give here arguments in support of our assumption of electron temperatures close to the value found for a stable ionospheric E-region.

We take first the equation for the electron temperature variation (Gurevich, 1978; Stenflo, 1985; St.-Maurice and Kissack, 2000)

$$
\begin{aligned}
& n \frac{\partial T_{e}}{\partial t}+n \mathbf{U} \cdot \nabla T_{e}+\nabla \cdot \mathbf{g}+\frac{2}{3} n T_{e} \nabla \cdot \mathbf{U}= \\
& \quad-n \delta_{e} v_{e}\left(T_{e}-T_{0}\right)+Q(\mathbf{r}, t)
\end{aligned}
$$

where $Q$ is an energy density input, which is for the moment unspecified. We have

$\mathbf{g}=-n \mathbf{\Xi}_{e} \cdot \nabla T_{e}$

being the heat flux density, with $\Xi_{e}$ being the electron thermal conductivity tensor

$$
\Xi_{e}=\frac{5}{3} \frac{T_{e}}{m}\left\{\begin{array}{ccc}
\frac{v_{e}}{\Omega_{c e}^{2}+v_{e}^{2}} & \frac{-\Omega_{c e}}{\Omega_{c e}^{2}+v_{e}^{2}} & 0 \\
\frac{\Omega_{c e}}{\Omega_{c e}^{2}+v_{e}^{2}} & \frac{v_{e}}{\Omega_{c e}^{2}+v_{e}^{2}} & 0 \\
0 & 0 & \frac{1}{v_{e}}
\end{array}\right\} .
$$

We ignore electron-ion collisions and assume for simplicity that all collisions with neutrals are "simple", i.e. that the cross section varies inversely proportional to the velocity, so that we can take the collision frequency $\nu_{e}$ to be a constant. The quantity $\delta_{e} \approx 2 m / M$ determines the energy loss of an electron per collision.

The steady state electron drift $\mathbf{U}(\mathbf{r})$ can be determined by

$$
\begin{aligned}
\mathbf{U}(\mathbf{r}) & \cdot \nabla \mathbf{U}(\mathbf{r})=-\frac{e}{m}(\mathbf{E}(\mathbf{r})+\mathbf{U}(\mathbf{r}) \times \mathbf{B}) \\
- & \frac{T_{e}(\mathbf{r})}{m n(\mathbf{r})} \nabla n(\mathbf{r})-\frac{1}{m} \nabla T_{e}(\mathbf{r})-v_{e}(\mathbf{r}) \mathbf{U}(\mathbf{r}),
\end{aligned}
$$

where we can often assume the left side to be small or vanishing. The B-parallel component is particularly simple,

$U_{\|}=-\frac{e}{m v_{e}} E_{\|}-\frac{T_{e}}{m n v_{e}} \frac{\partial}{\partial z} n-\frac{1}{m v_{e}} \frac{\partial}{\partial z} T_{e}$.

The basic element in the following arguments is that the expression Eq. (A1) is not complete before the boundary conditions have been specified. With the electrojet being $\sim 10 \mathrm{~km}$ in the vertical direction, it may seem plausible that it is safe to assume it to be infinite, but we have to take into account that the electron thermal conductivity is very large, $\Xi_{\|} \sim T_{e} /\left(v_{e} m\right)$, along the magnetic field lines. This conductivity has to "compete" with an electron cooling term, which is rather small because of the smallness of $\delta_{e}$. 
To substantiate the discussion we assume the magnetic field lines to be vertical for our case, and write the vertical component of Eq. (A1) for steady state horizontally stratified conditions, with no vertical electric field $E_{\|}=0$. We might assume that the electron temperature variations possibly induced by the plasma instability vary on a smaller length scale than the plasma density variations, so we retain only the part of $U_{\|}$that originates from the temperature gradient

$$
\begin{gathered}
\frac{d}{d z}\left(n \frac{5}{3} \frac{T_{e}}{m v_{e}} \frac{d}{d z} T_{e}\right)+\frac{n}{m v_{e}}\left(\frac{d T_{e}}{d z}\right)^{2}+\frac{2}{3} \frac{n T_{e}}{m v_{e}} \frac{d^{2}}{d z^{2}} T_{e}= \\
n \delta_{e} v_{e}\left(T_{e}-T_{0}(z)\right)-Q(z, t)
\end{gathered}
$$

assuming that also $Q$ is horizontally stratified. The $\mathbf{E} \times \mathbf{B}$-drift of the electrons does not enter here, since it appears in the expressions for the horizontal coordinates.

We now make an estimate for the electron temperature, by use of Eq. (A4). The electron temperature will have a maximum value somewhere in the electrojet, $T_{e m}$, at $z=z_{m}$, where $d T_{e} /\left.d z\right|_{z_{m}}=0$. We then have

$$
\left.n_{m} \frac{7}{3} \frac{T_{e m}}{m v_{e}} \frac{d^{2}}{d z^{2}} T_{e}\right|_{z_{m}}=n_{m} \delta_{e} v_{e}\left(T_{e m}-T_{0}\left(z_{m}\right)\right)-Q\left(z_{m}\right)
$$

As an estimate we make a local parabolic approximation for the temperature variation and take $d^{2} T_{e} / d z^{2} \approx$ $-\left(T_{e m}-T_{0}\left(z_{m}\right)\right) / \mathcal{L}^{2}$, where $\mathcal{L}$ is a characteristic length scale. In our case we can take $\mathcal{L} \sim 1-5 \mathrm{~km}$. We can estimate $T_{e} /\left(m v_{e}^{2}\right) \approx \ell_{c}^{2}$, where $\ell_{c}$ is the mean free path for collisions. We then have the estimate

$\frac{7}{3} \frac{\ell_{c}^{2}}{\delta_{e} \mathcal{L}^{2}}\left(T_{e m}-T_{0}\left(z_{m}\right)\right) \approx\left(T_{0}\left(z_{m}\right)-T_{e m}\right)+\frac{Q\left(z_{m}\right)}{n_{m} \delta_{e} v_{e}}$.

Although it is not apparent by the notation, this expression is nonlinear in $T_{m}$, because of the temperature dependence of $\ell_{c}$. With an effective average ion mass of 27 proton masses, we can estimate $\delta_{e} \approx 3.6 \times 10^{-5} \ll 1$.

Basically two different cases can be found: one where the left side is negligible and one where it dominates the first term on the right hand side, the controlling parameter being $\ell_{c}^{2} /\left(\delta_{e} \mathcal{L}^{2}\right)$. If we take a typical mean free path to be $\ell_{c} \approx 10 \mathrm{~m}$, we find the left side to be approximately 10 times larger that the other term, i.e. thermal conduction to the surroundings dominate for the conditions relevant for the ROSE4 rocket. This estimate of course depends on the actual parameter values, and for a detailed analysis it might be best to solve Eq. (A4) numerically with proper models for the altitude dependencies of the parameters. Even when the two terms in Eq. (A6) are comparable, we need approximately twice the energy deposition from the instability into the electrons, as compared to the infinite plasma model, in order to reach the same electron temperature.

One interesting aspect of the present discussion is that it indicates a basic difference between the equatorial and polar electrojets in this respect. For the equatorial case where we might assume the magnetic field to be horizontal, we have to take not the mean free path but the electron Larmor radius, $r_{e L} \ll \ell_{c}$, and we can for most relevant cases ignore the thermal conductivity to the $\mathrm{D}$ - and F-regions. For $B \approx 50 \mu \mathrm{T}$ and $T_{e} \approx 500 \mathrm{~K}$, we have $r_{e L} \approx 0.01 \mathrm{~m}$. The electron thermal conductivity is generally poor in the direction perpendicular to a magnetic field. These simple arguments indicate that there might be interesting physics to be learned by comparing details of observations of the saturated stage of these ionospheric instabilities from the equatorial and polar electrojets. For the present applications we find it justified to use a model with an electron temperature given by the standard electrojet values and ignore the possibility for an enhancement of $T_{e}$ by the instability.

The analysis of the present section has direct relevance for some laboratory studies of cross-field instabilities. In a Q-machine experiment, for instance, we can in general expect the electron temperature to be given as the hot-plate temperature due to the high electron thermal conductivity along magnetic field lines and also here ignore anomalous electron heating.

\section{Appendix B}

\section{A simple model for synthetic data}

We have previously commented upon a simple model for providing a set of synthetic data (Pécseli and Trulsen, 1993; Krane et al., 2000). Basically, the idea is to make a random superposition of some prescribed, deterministic space-time varying pulses. The randomness of the signal is then solely due to the statistical distributions of the reference time and position of each pulse. By the prescribed time variation we can model a growth due to the linear instability and a subsequent saturation and decline. While a structure is damping and vanishing at some position, other pulses are created at different positions. The model of course allows for a distribution of different structures, with prescribed densities. Let for instance a basic structure be given for the electrostatic potential as $\phi(\mathbf{r}, t)$. The space time varying signal is then constructed as

$$
\Phi(\mathbf{r}, t)=\sum_{j=1}^{N} \sum_{\ell=1}^{N} \phi\left(\mathbf{r}-\mathbf{r}_{j}, t-t_{\ell}\right),
$$

assuming $N$ pulses, and $\mathbf{r}_{j}$ being a pulse position uniformly distributed over a large volume while $t_{\ell}$ is similarly a pulse reference time uniformly distributed over a long time interval. If several pulse types are invoked, there will be an additional sum over the pulse types, given their individual statistical weights. It is now a simple matter (Pécseli, 2000; Krane et al., 2000) to obtain the correlation function for the signal as 


$$
\begin{aligned}
& \langle\Phi(\xi, \tau) \Phi(\xi+\mathbf{r}, \tau+t)\rangle= \\
& \mu \int_{-\infty}^{\infty} \iiint_{-\infty}^{\infty} \phi(\xi, \tau) \phi(\xi-\mathbf{r}, \tau-t) d \xi^{3} d \tau,
\end{aligned}
$$

where we assumed the volume and time intervals to be so large that we can let the integration limits be infinite. The density of pulses is introduced as $\mu$. The expression (B2) gives the correlation function for the space-time varying electrostatic potential in the model ionosphere. To obtain a model for the signal as detected by the rocket probes, we insert a probe positions as functions of time in Eq. (B1).

We have a basic choice in selecting either a pulse, or "blob" like basic structure (St.-Maurice and Hamza, 2001) or alternatively an oscillatory wave-like structure. The important point is here that only an oscillatory structure will be compatible with the observed auto-cross correlation functions, as found in Fig. 4: this figure shows crosscorrelations, but for the case where the time-delay vanishes, it reproduces an auto-correlation. Evidently: if the basic structure $\phi$ is oscillatory, so is the correlation function (B2).

Acknowledgements. Parts of the present work were carried out under the project "Turbulence in Fluids and Plasmas", conducted at the Centre for Advanced Study (CAS) in Oslo in 2004/2005. We thank the staff there for providing us a unique environment for the work. The Rocket and Scatter Experiment (ROSE) was performed in the framework of the German national sounding rocket program with international participation. It was primarily funded by the Bundesministerium für Forschung und Technologie (BMFT) and was managed by the Deutsche Gesellschaft für Luft- und Raumfahrt (DLR). The work by L. Dyrud and M. Oppenheim was partially supported by U.S. National Science Foundation Grant ATM-0442075."

Topical Editor M. Pinnock thanks L. Lyons and another referee for their help in evaluating this paper.

\section{References}

Alport, M. J., D’Angelo, N., and Pécseli, H. L.: A laboratory experiment on EM backscatter from Farley-Buneman and gradient drift waves, J. Geophys. Res., 86, 7694-7702, 1981.

Balsley, B. B.: Some characteristics of the non-two-stream irregularities in the equatorial electrojet, J. Geophys. Res., 74, 2333 $2347,1969$.

Balsley, B. B. and Farley, D. T.: Radar observations of twodimensional turbulence in the equatorial electrojet, J. Geophys. Res., 78, 7471-7479, 1973.

Bendat, J. S.: Principles and Applications of Random Noise Theory, John-Wiley \& Sons, New York, 1958.

Buneman, O.: Excitation of field aligned sound waves by electron streams, Phys. Rev. Lett., 10, 285-287, 1963.

D’Angelo, N., Pécseli, H. L., and Petersen, P. I.: Farley instability - a laboratory test, J. Geophys. Res., 79, 4747-4751, 1974.

Dimant, Y. S. and Oppenheim, M. M.: Ion thermal effects on Eregion instabilities: linear theory, J. Atmos. Solar-Terr. Phys., 66, 1639-1654, 2004.
Farley, D. T.: Two-stream plasma instability as a source of irregularities in the ionosphere, Phys. Rev. Lett., 10, 279-282, 1963.

Fejer, B. G., Providakes, J., and Farley, D. T.: Theory of plasma waves in the auroral E region, J. Geophys. Res., 89, 7487-7494, 1984.

Friker, A. and Lübken, F.-J.: Neutral air density and temperature measurements by the TOTAL instrument onboard the ROSE payloads, J. Atmos. Terr. Phys., 54, 693-701, 1992.

Gurevich, A. V.: Nonlinear Phenomena in the Ionosphere, vol. 10 of Physics and Chemistry in Space, Springer, New York, 1978.

Gurevich, A. V., Borisov, N. D., and Zybin, K. P.: Ionospheric turbulence induced in the lower parts of the E region by the turbulence in the neutral atmosphere, J. Geophys. Res., 102, 379-388, 1997.

Hallatschek, K. and Diamond, P. H.: Modulational instability of drift waves, New J. Phys., 5, 29.1-29.9, 2003.

Hamza, A. M. and St.-Maurice, J. P.: A fully self-consistent fluid theory of anomalous transport in Farley-Buneman turbulence, J. Geophys. Res., 100, 9653-9668, 1995.

Hinze, J. O.: Turbulence, 2 ed., McGraw Hill, New York, 1975.

Iranpour, K., Pécseli, H. L., Trulsen, J., Bahnsen, A., Primdahl, F., and Rinnert, K.: waves in the ionospheric E region, Ann. Geophys., 15, 878-889, 1997.

John, P. I. and Saxena, Y. C.: Observation of Farley-Buneman instability in laboratory plasma, Geophys. Res. Lett., 2, 251-254, 1975.

Kelley, M. C. and Mozer, F. S.: Electric field and plasma density oscillations due to the high-frequency Hall current two-stream instability in the Auroral E region, J. Geophys. Res., 78, 2214 2221, 1973.

Kohl, H., Nielsen, E., Rinnert, K., and Schlegel, K.: EISCAT results during the ROSE campaign and comparison with STARE measurements, J. Atmos. Terr. Phys., 54, 733-739, 1992.

Krane, B., Pécseli, H. L., Trulsen, J., and Primdahl, F.: Spectral properties of low-frequency electrostatic waves in the ionospheric E region, J. Geophys. Res., 105, 10 585-10 601, 2000.

Larsen, Y., Hanssen, A., Krane, B., Pécseli, H. L., and Trulsen, J.: Time-resolved statistical analysis of nonlinear electrostatic fluctuations in the ionospheric E region, J. Geophys. Res., 107, 1005, doi:10.1029/2001JA900125, 2002.

Leslie, D. C.: Developments in the Theory of Turbulence, Oxford University Press, Oxford, 1973.

Mallat, S.: A Wavelet Tour of Signal Processing, Academic Press, San Diego, California, 1998.

Mikkelsen, T. and Pécseli, H. L.: Strong turbulence in partially ionized plasmas, Phys. Lett. A, 77, 159-162, 1980.

Oppenheim, M. and Otani, N.: Spectral characteristics of the Farley-Buneman instability: Simulations versus observations, J. Geophys. Res., 101, 24 573-24 582, 1996.

Oppenheim, M., Otani, N., and Ronchi, C.: Hybrid simulations of the saturated Farley-Buneman instability in the ionosphere, Geophys. Res. Lett., 22, 353-356, 1995.

Oppenheim, M. M. and Dimant, Y. S.: Ion thermal effects on Eregion instabilities: 2D kinetic simulations, J. Atmos. Solar-Terr. Phys., 66, 1655-1668, 2004.

Oppenheim, M. M., Dyrud, L. P., and vom Endt, A. F.: Plasma instabilities in meteor trails: 2-D simulation studies, J. Geophys. Res., 108, 1064, doi:10.1029/2002JA009549, 2003.

Pécseli, H. L.: Fluctuations in Physical Systems, Cambridge Uni- 
versity Press, Cambridge, UK, 2000.

Pécseli, H. L. and Trulsen, J.: On the interpretation of experimental methods for investigating nonlinear wave phenomena, Plasma Phys. Contr. Fusion, 35, 1701-1715, 1993.

Pécseli, H. L., Primdahl, F., and Bahnsen, A.: Low-frequency electrostatic turbulence in the polar cap E region, J. Geophys. Res., 94, 5337-5349, 1989.

Pécseli, H. L., Trulsen, J., Primdahl, F., and Bahnsen, A.: Propagation and nonlinear interaction of low-frequency electrostatic waves in the polar cap E region, J. Geophys. Res., 98, 16031612, 1993.

Pfaff, R. F., Kelley, M. C., Fejer, B. G., Kudeki, E., Carlson, C. W., Pedersen, A., and Hausler, B.: Electric field and plasma density measurements in the Auroral electrojet, J. Geophys. Res., 89, 236-244, 1984.

Primdahl, F.: Polar ionospheric E region plasma wave stabilization and electron heating by wave-induced enhancements of the electron collision frequency, Phys. Scripta, 33, 187-191, 1986.

Primdahl, F. and Bahnsen, A.: Auroral E-region diagnosis by means of nonlinearly stabilized plasma waves, Ann. Geophys., 3, 5762, 1985.

Rinnert, K.: Plasma waves observed in the Auroral E region ROSE campaign, J. Atmos. Terr. Phys., 54, 683-692, 1992.

Rogister, A.: Nonlinear theory of cross-field instability with applications to the equatorial electrojet, J. Geophys. Res., 77, 29752981, 1972.

Rogister, A. and D'Angelo, N.: Type II irregularities in the equatorial electrojet, J. Geophys. Res., 75, 3879-3887, 1970.

Rose, G., Schlegel, K., Rinnert, K., et al.: The ROSE project scientific objectives and discussion of 1st results, J. Atmos. Terr. Phys., 54, 657-667, 1992.
Schlegel, K.: The influence of metallic ions on the plasma instabilities in the high-latitude E region, Radio Science, 20, 740-744, 1985.

Schlegel, K.: Measurements of electron density fluctuations during the ROSE rocket flights, J. Atmos. Terr. Phys., 54, 715-723, 1992.

Schlegel, K. and Gurevich, A. V.: Radar backscatter from plasma irregularities of the lower $\mathrm{E}$ region induced by neutral turbulene, Ann. Geophys., 15, 870-877, 1997.

Shkarofsky, I. P.: Turbulence in Fluids and Plasmas, chap. 21 "Analytic forms for decaying turbulence functions", Polytechnic Press, Brooklyn, N.Y., 1969.

St.-Maurice, J.-P.: A nonlocal theory of the high-latitude FarleyBuneman instability, J. Geophys. Res., 90, 5211-5225, 1985.

St.-Maurice, J.-P. and Hamza, A. M.: A new nonlinear approach to the theory of E region irregularities, J. Geophys. Res., 106, 1751-1759, 2001.

St.-Maurice, J.-P. and Kissack, R. S.: The role played by thermal feedback in heated Farley-Buneman waves at high latitudes, Ann. Geophys., 18, 532-546, 2000.

Stenflo, L.: Parametric excitation of collisional modes in the highlatitude ionosphere, J. Geophys. Res., 90, 5355-5356, 1985.

Sudan, R. N.: Unified theory of type-I and type-II irregularities in the Equatorial electrojet, J. Geophys. Res., 88, 4853-4860, 1983.

Tchen, C. M.: Repeated cascade theory of turbulence in an inhomogeneous plasma, Phys. Rev. A, 8, 500-514, 1973.

Thrane, E. V., Grandal, B., Flå, T., and Brekke, A.: Fine structure in the ionospheric D-region, Nature, 292, 221-223, 1981.

Wernik, A. W.: Methods of data analysis for resolving nonlinear phenomena, in: Modern Ionospheric Physics, edited by: Kohl, H., Rüster, R. and Schlegel, K., 321-345, European Geophysical Society, Katlenburg-Lindau, Germany, 1996. 